\title{
Social Relationships and Its Association With Affective Symptoms of Women With Breast Cancer: a Scoping Review
}

\author{
Yesol Yang ( $\nabla$ yang.6310@osu.edu ) \\ Ohio State University Comprehensive Cancer Center-James \\ Yufen Lin \\ Emory University \\ Grace Sikapokoo \\ Ohio University \\ Se Hee Min \\ Duke University Medical Library \\ Nicole Caviness-Ashe \\ Duke University \\ Jing Zhang \\ The Ohio State University \\ Leila Ledbetter \\ Duke University Medical Library \\ Timiya Nolan \\ The Ohio State University
}

\section{Research Article}

Keywords: Interpersonal relations, affective symptoms breast neoplasms

Posted Date: November 17th, 2021

DOI: https://doi.org/10.21203/rs.3.rs-1005008/v1

License: (c) (i) This work is licensed under a Creative Commons Attribution 4.0 International License. Read Full License 


\section{Abstract}

Background: Problems in affective and cognitive functioning are among the most common concurrent symptoms that breast cancer patients report. Social relationships may provide some explanations of the clinical variability in affectivecognitive symptoms. Evidence suggests that social relationships (functional and structural aspects) can be associated with patients' affective-cognitive symptoms; however, such an association has not been well studied in the context of breast cancer. The purpose of this scoping review was to address the following question: What social relationships are associated with affective-cognitive symptoms of women with breast cancer? This scoping review used the framework proposed by Arksey and O'Malley and PRISMA-Sc. Extracted data included research aims, design, sample, type and measures of social relationships (functional and structural), and the association between social relationships and affective-cognitive symptoms.

Results: Of sixty-five included studies, none of them focused on cognitive symptoms of breast cancer patients; thus, in this review, we focused on only the affective symptoms of breast cancer patients and their association with patients' both aspects of social relationships.

Conclusion: Our findings reveal that positive social relationships benefit in mitigating affective symptoms of women with breast cancer. Thus, health care providers need to educate patients about the importance of building solid social relationships and encourage them to participate in a supportive network of friends and family members.

\section{Background}

With advances in medical treatments, breast cancer mortality rates have steadily declined in recent years, resulting in an increase in 5-year survival rates.(1) According to the report from American Cancer Society in 2017, the overall survival rates have increased from $68-89 \%$ for White women and from $55-81 \%$ for Black women.(2) Resultantly, cancer is no longer viewed as an incurable acute disease. Instead, it follows the trajectories of chronic diseases that is characterized by periods of remission and exacerbation of symptoms.(3) Women with breast cancer often experience symptoms that co-occur (i.e., symptom clusters) during the disease trajectory.(3) For example, patients experience affective and cognitive problems (symptoms) concurrently. The co-occurrence of these symptoms is called a psychoneurological symptom cluster.(4) Further, these two symptoms within a psychoneurological cluster are strongly related to each other. $(4,5)$

Problems in affective and cognitive functioning are among the most common concurrent symptoms that breast cancer patients report. $(4,5)$ Cognitive symptoms include changes in memory, concentration, processing speed, executive function, and language, whereas affective symptoms include mood changes such as anxiety and depression. $(4,5)$ Factors that contribute to affective-cognitive (i.e., psychoneurological) symptoms were identified as stress, hypothalamic-pituitary-adrenocortical axis dysfunction, cytokine dysregulation, telomere shortening, or DNA damage(4); however, these factors do not sufficiently explain the variability in cognitive-affective symptoms. For example, some patients have reported persistent and high levels of mood disturbance and cognitive impairment for several years or more following cancer treatment.(6) This finding suggests a need to investigate other potential factors that can explain their clinical variabilities.

Social relationships may provide some explanations of the clinical variability in affective-cognitive symptoms. Social relationships can be characterized as aspects, structural and functional. The structural aspect reflects the size, scope, and connectedness of social relations (e.g., social integration), while the functional aspect covers the interpersonal interaction within the structure of the social relations (e.g., social support).(7) Both aspects of social relationships can influence patients' affective and cognitive symptoms. Recent systematic reviews have reported that the older 
populations showed a greater decline in their cognition when their social relationship was functionally and structurally poor. $(8,9)$ Additionally, patients who had greater social support and cohesive relationships with their family members showed fewer depressive symptoms. $(10,11)$

Evidence suggests that social relationships can be associated with fewer patients' affective-cognitive symptoms. One study has reported that breast cancer survivors demonstrated higher levels of depressive symptoms over the trajectory of their illness when they received lower levels of social support.(12) In addition, those with less social support showed cytokine dysregulation which is recognized as a contributing factor for cognitive symptoms. $(12,13)$ Other studies have reported that social relationships play an important role in the protection against affective symptoms(14, 15), ultimately improving survival outcomes of breast cancer patients.(15) Social relationships (functional and structural aspects) appear to be associated with fewer affective-cognitive symptoms in breast cancer patients; however, such association has not been well studied.

\section{Purpose}

The purpose of this scoping review was to address the following question: What social relationships are associated with affective-cognitive symptoms of women with breast cancer? This will lay the foundation for studies that explicate the mechanism of affective-cognitive symptoms in breast cancer patients. This understanding will also allow clinicians to identify patients more precisely at risk for affective-cognitive symptoms associated with social relationships and will contribute to the development of strategies to prevent and manage these symptoms.

\section{Methods}

We followed the five methodological stages of scoping review developed by Arksey and O'Malley(16). This review was conducted based on the following stages: 1) identifying the research question, 2) identifying relevant studies, 3) selecting studies, 4) charting the data, and 5) collating, summarizing, and reporting the results. We reported using the Preferred Reporting Items for Systematic Reviews and Meta-Analyses extension for Scoping Reviews (PRISMA-ScR). (17) Figure 1 presents study selection by flowchart as per PRISMA guidelines.

Stage 1. Identifying research questions

We identified a research question using the PCO model(16): "What social relationships ("C", concept) are associated with affective-cognitive symptoms ("O", outcome) of women with breast cancer ("P", population)?" We limited our study population of women with breast cancer aged 18 years and above because of different trajectories and manifestations of cognitive symptoms that children with cancer show compared with adults.(18) Table 1 describes eligibility criteria for the studies that were included in this scoping review. 
Table 1

Inclusion criteria and exclusion criteria

\section{Inclusion criteria}

Type of
publication

Time
frame

\section{Language English}

$\begin{array}{ll}\begin{array}{l}\text { Study } \\ \text { population }\end{array} & \text { Adult (18 years or older) women with breast cancer who are undergoing active or completed cancer } \\ \text { treatments }\end{array}$ Outcome Studies that assessed either or both affective (e.g., anxiety, depression) and cognitive symptoms (e.g., of interest cognitive dysfunction/impairment/decline or neurocognitive disorder)

\section{Exclusion criteria}

Studies that did not assess the association between social relationships and patients' affective-cognitive symptom.

Studies that were explicitly about individuals with cognitive deficits attributed to non-cancer causes such as psychiatric or neurological illness, dementia, stroke, brain injury or delirium.

Stage 2. Identifying relevant studies

We developed relevant search terms in collaboration with a librarian included a mix of keywords and database specific subject headings representing women, breast cancer, affective symptoms and social relationships. The search was translated and conducted by a medical research librarian on September 14, 2020 using four databases: MEDLINE (PubMed), Embase (Elsevier), PsycINFO (EBSCOhost), and Web of Science (Clarivate). Editorials, letters, and comments were excluded, as were animal-only studies and studies involving pediatric populations. Reproducible search strategies for all databases can be found in Appendix A. We reviewed the results for existing review articles and determined that no review articles currently exist on our topic.

Stage 3. Study selection

The search identified a total of 4,793 references that were imported into Covidence, a systematic review screening tool (Covidence systematic review software, Veritas Health Innovation, Melbourne, Australia. Available at www.covidence.org). Duplicate citations $(n=1,348)$ were automatically identified and removed by Covidence. The software ensures that two reviewers independently screened a total of 3,445 references by title and abstract. Studies were excluded if they did not clearly meet inclusion criteria, and of those, 3,291 references were deemed irrelevant and excluded. Upon the completion of screening titles and abstracts, any disagreements were resolved by discussion. One hundred thirty-eight citations were identified for full text assessment. At the full text review stage, articles were independently read by two different members of team (YY, YL, GS). During the full-text review, each study was reviewed independently to determine the final sample. Full-text studies that did not meet the inclusion criteria were excluded, and the reasons for exclusion were noted. Disagreement between the team members were resolved through discussion. 65 articles were confirmed to be included in the final set for data extraction (Figure 1).

Stage 4. Charting the data

Our team developed a data extraction tool and determined which data should be extracted from studies to answer the research question. Two team members (YY, GS) independently piloted data abstraction from the first fifteen included 
studies using the data charting form. Then, they discussed the process and their results to confirm whether their approaches to data extraction were consistent. Questions arising when piloting the extract data form were discussed with the other team members ( $\mathrm{YL}, \mathrm{TN})$. After piloting the form, two team members ( $\mathrm{YY}, \mathrm{GS})$ independently recorded the following data from selected studies on the data charting form: 1) authors, 2) country of study, 3) year of publication, 4) study design, 5) sample characteristics (sample size, age, and type of cancer treatment), 6) type of social relationships 7) affective-cognitive symptoms and measurements, and 8) key findings (the association between social relationships and affective-cognitive symptoms).

Stage 5. Collating, summarizing and reporting the results

Our team collated, summarized, and reported all data obtained in stage 4 to map the knowledge on social relationships associated with affective-cognitive symptoms of adult women with breast cancer. The studies in the final sample were tabulated based on social relationships (e.g., functional or structural aspect of social relations). A table for the final sample was created and included the information on authors, years of publication, country of study, study population, type of social relationships, measures of affective-cognitive symptoms, and the association between social relationships and affective-cognitive symptoms. Verification of data accuracy was impudently conducted by six research team members (YY, YL, GS, SM, NC, JZ).

\section{Results}

\subsection{Study characteristics}

Table 2 includes sixty-five studies that met the inclusion criteria. The reviewed studies were conducted in 22 countries with majority conducted in the US $(n=30)$ and Canada $(n=4)$. Of 65 studies, 31 were cross-sectional, 4 were randomized controlled trials, 9 were longitudinal, and 21 were a secondary analysis from a cross-sectional, longitudinal, or multipleinstitutional cohort study. The sample size of dyadic studies (included both patients and their spouses/partners/family caregivers) ranging from 92 to 470, and the sample size of the remaining 60 non-dyadic studies ranged from 25 to 2235 patients. The mean age of patients who participated in this study ranged from 36.7 to 66.7 years old. Also, participants in the published studies from the US were White (Caucasian), followed by Black (African American), Latina or Asian. Of the included studies, three dealt with patients living with metastatic/advanced breast cancer. Additionally, cancer treatments that patients received were varied including chemotherapy, surgery, hormone, radiation, and targeted therapy. 
Table 2

Study Characteristics

\begin{tabular}{|c|c|c|c|c|c|}
\hline \multirow{2}{*}{$\begin{array}{l}\text { Author (year), } \\
\text { Country }\end{array}$} & \multirow{2}{*}{$\begin{array}{l}\text { Study } \\
\text { design }\end{array}$} & \multicolumn{4}{|c|}{ Sample characteristics } \\
\hline & & $\mathbf{N}$ & Race/ethnicity & $\begin{array}{l}\text { Age } \\
\text { (mean, SD) }\end{array}$ & Tx \\
\hline $\begin{array}{l}\text { Roberts et al.,1994 } \\
\text { (USA) }\end{array}$ & $\begin{array}{l}\text { Secondary } \\
\text { analysis }\end{array}$ & $\begin{array}{l}135 \text { women } \\
\text { with breast } \\
\text { cancer }\end{array}$ & Not reported & $\begin{array}{l}56.2 \\
(S D=11.9)\end{array}$ & Surgery $(100 \%)$ \\
\hline $\begin{array}{l}\text { Neuling et al., } 1988 \\
\text { (Australia) }\end{array}$ & Longitudinal & $\begin{array}{l}58 \text { women } \\
\text { with breast } \\
\text { cancer }\end{array}$ & Not reported & Median=54 & Surgery $(100 \%)$ \\
\hline $\begin{array}{l}\text { Koopman et al., } \\
1998 \text { (USA) }\end{array}$ & $\begin{array}{l}\text { Cross- } \\
\text { sectional }\end{array}$ & $\begin{array}{l}102 \text { women } \\
\text { with } \\
\text { metastatic } \\
\text { and/or } \\
\text { recurrent } \\
\text { breast cancer }\end{array}$ & $\begin{array}{l}\text { Caucasian (88.2\%); } \\
\text { Asian-American } \\
(4.9 \%) ; \text { African- } \\
\text { American (1\%); } \\
\text { Hispanic/Latina } \\
(2 \%) ; \text { Native } \\
\text { American }(2 \%) ; \\
\text { Other (2\%) }\end{array}$ & $\begin{array}{l}53.1 \\
(S D=10.8)\end{array}$ & $\begin{array}{l}\text { CTx (52\%); Hormone } \\
(76.5 \%)\end{array}$ \\
\hline $\begin{array}{l}\text { Lee et al., } \\
2004 \text { (Korea) }\end{array}$ & $\begin{array}{l}\text { Cross- } \\
\text { sectional }\end{array}$ & $\begin{array}{l}134 \text { women } \\
\text { receiving } \\
\text { chemotherapy } \\
\text { for breast } \\
\text { cancer }\end{array}$ & Korean (100\%) & $\begin{array}{l}45.29 \\
(S D=8.75)\end{array}$ & CTx $(100 \%)$ \\
\hline $\begin{array}{l}\text { Maly et al., } \\
2005 \text { (USA) }\end{array}$ & $\begin{array}{l}\text { Cross- } \\
\text { sectional }\end{array}$ & $\begin{array}{l}222 \text { women } \\
\text { with newly } \\
\text { diagnosed } \\
\text { breast cancer }\end{array}$ & $\begin{array}{l}\text { White }(64 \%) ; \\
\text { African-American } \\
(12 \%) ; \text { Latina } \\
(23 \%) ; \text { Other }(1 \%)\end{array}$ & $\begin{array}{l}66.7 \\
(S D=7.9)\end{array}$ & $\begin{array}{l}\text { Surgery (31.5\%); RTX } \\
(40 \%) ; \text { CTX (37.4\%) }\end{array}$ \\
\hline $\begin{array}{l}\text { Palesh et al., } 2006 \\
\text { (USA) }\end{array}$ & $\begin{array}{l}\text { Cross- } \\
\text { sectional }\end{array}$ & $\begin{array}{l}82 \text { women } \\
\text { recently } \\
\text { diagnosed } \\
\text { with breast } \\
\text { cancer stage } \\
\text { 0-III }\end{array}$ & Not reported & $\begin{array}{l}57.4 \\
(S D=11.5)\end{array}$ & $\begin{array}{l}\text { Surgery } \\
\text { (mastectomy, 43\%; } \\
\text { lumpectomy, } \\
79.3 \%) ; \\
\text { CTx }(50 \%) ; R T x \\
(59.8 \%) ; \\
\text { Hormone }(42.7 \%)\end{array}$ \\
\hline $\begin{array}{l}\text { Friedman et al., } \\
2006 \text { (USA) }\end{array}$ & $\begin{array}{l}\text { Cross- } \\
\text { sectional }\end{array}$ & $\begin{array}{l}81 \text { women } \\
\text { with breast } \\
\text { cancer }\end{array}$ & $\begin{array}{l}\text { African-American; } \\
\text { Hispanic; } \\
\text { Caucasian (\% not } \\
\text { reported) }\end{array}$ & $\begin{array}{l}52 \\
(S D=10.2)\end{array}$ & $\begin{array}{l}\text { Surgery (74\%); CTx } \\
(88.9 \%)\end{array}$ \\
\hline $\begin{array}{l}\text { Porter et al., } 2006 \\
\text { (USA) }\end{array}$ & $\begin{array}{l}\text { Secondary } \\
\text { analysis }\end{array}$ & $\begin{array}{l}524 \text { women } \\
\text { with breast } \\
\text { cancer }\end{array}$ & $\begin{array}{l}\text { White }(70.6 \%) \text {; } \\
\text { African-American } \\
(29.4 \%)\end{array}$ & $\begin{array}{l}64.5 \\
(S D=8.9)\end{array}$ & $\begin{array}{l}\text { Surgery }(98.6 \%) ; C T x \\
(23 \%) ; R T x(27 \%) ; \\
\text { Hormone }(28 \%)\end{array}$ \\
\hline $\begin{array}{l}\text { Kim \& Morrow, } \\
2007 \text { (USA) }\end{array}$ & $\begin{array}{l}\text { Secondary } \\
\text { analysis }\end{array}$ & $\begin{array}{l}539 \text { women } \\
\text { with breast } \\
\text { cancer }\end{array}$ & Caucasian (94\%) & 51 & CTx $(100 \%)$ \\
\hline $\begin{array}{l}\text { Nausheen \& Kamal, } \\
2007 \text { (Pakistan) }\end{array}$ & $\begin{array}{l}\text { Cross- } \\
\text { sectional }\end{array}$ & $\begin{array}{l}82 \text { Pakistani } \\
\text { women with } \\
\text { breast cancer }\end{array}$ & Pakistan (100\%) & 42.5 & Surgery (90\%) \\
\hline
\end{tabular}




\begin{tabular}{|c|c|c|c|c|c|}
\hline \multirow{2}{*}{$\begin{array}{l}\text { Author (year), } \\
\text { Country }\end{array}$} & \multirow{2}{*}{$\begin{array}{l}\text { Study } \\
\text { design }\end{array}$} & \multicolumn{4}{|c|}{ Sample characteristics } \\
\hline & & $\mathbf{N}$ & Race/ethnicity & $\begin{array}{l}\text { Age } \\
\text { (mean, SD) }\end{array}$ & Tx \\
\hline $\begin{array}{l}\text { Von Ah \& Kang, } \\
2008 \text { (USA) }\end{array}$ & Longitudinal & $\begin{array}{l}49 \text { American } \\
\text { women with } \\
\text { newly breast } \\
\text { cancer stage } \\
0 \text {-III }\end{array}$ & $\begin{array}{l}\text { Caucasian (61\%); } \\
\text { African-American } \\
\text { (29\%); Asian- } \\
\text { American (4\%); } \\
\text { Hispanic-American } \\
\text { (2\%); Native } \\
\text { American (4\%) }\end{array}$ & $\begin{array}{l}52.3 \\
(\mathrm{SD}=9.6)\end{array}$ & $C T x+R T x(51 \%)$ \\
\hline $\begin{array}{l}\text { Gellaitry et al., } 2010 \\
\text { (UK) }\end{array}$ & RCT & $\begin{array}{l}80 \text { women } \\
\text { with breast } \\
\text { cancer } \\
\text { (n=38 } \\
\text { intervention } \\
\text { group) }\end{array}$ & Not reported & $\begin{array}{l}58.4 \\
(S D=10.8)\end{array}$ & $\begin{array}{l}\text { Surgery }(100 \%) ; C T x \\
(53 \%) ; R T x(100 \%) ; \\
\text { Hormone }(82 \%)\end{array}$ \\
\hline $\begin{array}{l}\text { Gorman et al., } 2010 \\
\text { (USA) }\end{array}$ & $\begin{array}{l}\text { Cross- } \\
\text { sectional }\end{array}$ & $\begin{array}{l}131 \text { women } \\
\text { with early- } \\
\text { stage breast } \\
\text { cancer }\end{array}$ & $\begin{array}{l}\text { White }(87.8 \%) \\
\text { Other (12.2\%) }\end{array}$ & $\begin{array}{l}36.7 \\
\text { (at } \\
\text { diagnosis) }\end{array}$ & $\begin{array}{l}C T x(88.6 \%) ; R T x \\
(55.7 \%)\end{array}$ \\
\hline $\begin{array}{l}\text { Hasson-Ohayon et } \\
\text { al., } 2010 \text { (Israel) }\end{array}$ & $\begin{array}{l}\text { Cross- } \\
\text { sectional }\end{array}$ & $\begin{array}{l}150 \text { dyads of } \\
\text { women with } \\
\text { breast cancer } \\
\text { stage III-IV } \\
\text { and their } \\
\text { spouses }\end{array}$ & Israel (100\%) & $\begin{array}{l}\text { Patients: } \\
53.15 \\
(S D=10.28)\end{array}$ & $\begin{array}{l}\text { Mostly not on active } \\
\text { treatment }\end{array}$ \\
\hline $\begin{array}{l}\text { Kim et al., } 2010 \\
\text { (USA) }\end{array}$ & $\begin{array}{l}\text { Cross- } \\
\text { sectional }\end{array}$ & $\begin{array}{l}231 \\
\text { undeserved } \\
\text { women with } \\
\text { breast cancer }\end{array}$ & $\begin{array}{l}\text { Caucasian }(62.3 \%) \text {; } \\
\text { African-American } \\
(35.9 \%) \text {; other } \\
\text { minorities }(1.7 \%)\end{array}$ & 51 & NR \\
\hline $\begin{array}{l}\text { Talley et al., } 2010 \\
\text { (USA) }\end{array}$ & $\begin{array}{l}\text { Secondary } \\
\text { analysis }\end{array}$ & $\begin{array}{l}163 \text { women } \\
\text { with breast } \\
\text { cancer }\end{array}$ & $\begin{array}{l}\text { White }(94.5 \%) \text {; } \\
\text { Black }(2.5 \%) ; \text { Other } \\
(1.8 \%)\end{array}$ & $\begin{array}{l}57.33 \\
(S D=11.22)\end{array}$ & NR \\
\hline $\begin{array}{l}\text { Cohen et al., } 2011 \\
\text { (Israel) }\end{array}$ & $\begin{array}{l}\text { Cross- } \\
\text { sectional }\end{array}$ & $\begin{array}{l}56 \text { women } \\
\text { with breast } \\
\text { cancer (stage } \\
\text { I-III) }\end{array}$ & Arabs $(100 \%)$ & $\begin{array}{l}50.6 \\
(S D=8.7)\end{array}$ & NR \\
\hline Hill et al., 2011 (UK) & Longitudinal & $\begin{array}{l}260 \text { women } \\
\text { with breast } \\
\text { cancer }\end{array}$ & Not reported & $\begin{array}{l}151 \\
\text { patients } \\
\text { aged 51-64 } \\
\text { years }\end{array}$ & Surgery (100\%) \\
\hline $\begin{array}{l}\text { Lee et al., } 2011 \\
\text { (Korea) }\end{array}$ & $\begin{array}{l}\text { Secondary } \\
\text { analysis }\end{array}$ & $\begin{array}{l}286 \text { women } \\
\text { with breast } \\
\text { cancer stage I- } \\
\text { III }\end{array}$ & Korean (100\%) & $\begin{array}{l}47 \\
(S D=10)\end{array}$ & $\begin{array}{l}\text { Breast Conserving } \\
\text { Surgery }(82.5 \%) ; \\
\text { Mastectomy } \\
(16.4 \%) ; \\
\text { CTx }(86.7 \%) ; \text { RTx } \\
(82.5 \%) ; \\
\text { Hormone }(82.2 \%)\end{array}$ \\
\hline $\begin{array}{l}\text { Liu et al., } 2011 \\
\text { (China) }\end{array}$ & $\begin{array}{l}\text { Cross- } \\
\text { sectional }\end{array}$ & $\begin{array}{l}401 \text { women } \\
\text { with breast } \\
\text { cancer }\end{array}$ & Chinese (100\%) & $\begin{array}{l}46.9 \\
(S D=10.1)\end{array}$ & NR \\
\hline
\end{tabular}




\begin{tabular}{|c|c|c|c|c|c|}
\hline \multirow{2}{*}{$\begin{array}{l}\text { Author (year), } \\
\text { Country }\end{array}$} & \multirow{2}{*}{$\begin{array}{l}\text { Study } \\
\text { design }\end{array}$} & \multicolumn{4}{|c|}{ Sample characteristics } \\
\hline & & $\mathbf{N}$ & Race/ethnicity & $\begin{array}{l}\text { Age } \\
\text { (mean, SD) }\end{array}$ & Tx \\
\hline $\begin{array}{l}\text { Boinon et al., } 2012 \\
\text { (France) }\end{array}$ & $\begin{array}{l}\text { Cross- } \\
\text { sectional }\end{array}$ & $\begin{array}{l}113 \text { women } \\
\text { with breast } \\
\text { cancer }\end{array}$ & Not reported & $\begin{array}{l}52.8 \\
(S D=10.17)\end{array}$ & Surgery (31\%) \\
\hline $\begin{array}{l}\text { Jones et al., } 2012 \\
\text { (Canada) }\end{array}$ & $\begin{array}{l}\text { Cross- } \\
\text { sectional }\end{array}$ & $\begin{array}{l}131 \text { women } \\
\text { with early- } \\
\text { stage breast } \\
\text { cancer }\end{array}$ & Not reported & $\begin{array}{l}54.6 \\
(S D=9.13)\end{array}$ & $\begin{array}{l}\text { Surgery }(96.9 \%) \\
\text { CTx }(57.3 \%) ; R T \\
(51.1 \%)\end{array}$ \\
\hline $\begin{array}{l}\text { Mallinckrodt et al., } \\
2012 \text { (USA) }\end{array}$ & Longitudinal & $\begin{array}{l}154 \text { women } \\
\text { with breast } \\
\text { cancer }\end{array}$ & $\begin{array}{l}\text { White non-Hispanic } \\
\text { ( } 97 \%) \text {; African- } \\
\text { American }(2.6 \%) \text {; } \\
\text { Hispanic }(0.6 \%)\end{array}$ & $\begin{array}{l}58.97 \\
(S D=12.33)\end{array}$ & $\begin{array}{l}\text { CTx+RTx (19\%); CTx } \\
(32 \%) ; \operatorname{RTx}(18 \%)\end{array}$ \\
\hline $\begin{array}{l}\text { Popoola \& } \\
\text { Adewuya, } 2012 \\
\text { (Nigeria) }\end{array}$ & $\begin{array}{l}\text { Cross- } \\
\text { sectional }\end{array}$ & $\begin{array}{l}124 \text { women } \\
\text { with breast } \\
\text { cancer }\end{array}$ & Nigerian (100\%) & NR & $\begin{array}{l}\text { Surgery }(9.7 \%) \\
\text { Surgery+CTx } \\
(39.5 \%) ; \\
\text { Surgery+CTx+RTx } \\
(50.8 \%)\end{array}$ \\
\hline $\begin{array}{l}\text { So et al., } 2013 \\
\text { (China) }\end{array}$ & $\begin{array}{l}\text { Secondary } \\
\text { analysis }\end{array}$ & $\begin{array}{l}279 \text { women } \\
\text { with breast } \\
\text { cancer }\end{array}$ & Chinese (100\%) & NR & NR \\
\hline $\begin{array}{l}\text { Waters et al., } 2013 \\
\text { (USA) }\end{array}$ & $\begin{array}{l}\text { Secondary } \\
\text { analysis }\end{array}$ & $\begin{array}{l}480 \text { women } \\
\text { with breast } \\
\text { cancer stage } \\
0 \text {-IIA }\end{array}$ & $\begin{array}{l}\text { White (81.5\%); non- } \\
\text { White (18.5\%) }\end{array}$ & $\begin{array}{l}58.3 \\
(S D=10.6)\end{array}$ & $\begin{array}{l}\text { Surgery }(100 \%) ; C T x \\
(24.8 \%) ; R T x(60 \%) ; \\
\text { Hormone }(51.3 \%)\end{array}$ \\
\hline $\begin{array}{l}\text { Yi \& Kim, } 2013 \\
\text { (Korea) }\end{array}$ & $\begin{array}{l}\text { Cross- } \\
\text { sectional }\end{array}$ & $\begin{array}{l}258 \text { Korean } \\
\text { women with } \\
\text { breast cancer }\end{array}$ & Korean (100\%) & $\begin{array}{l}47.45 \\
(S D=7.37)\end{array}$ & $\begin{array}{l}\text { Surgery }(98.8 \%) ; \\
\text { CTx }(83.3 \%) ; R T x \\
(55.4 \%) ; \text { Hormone } \\
(55 \%)\end{array}$ \\
\hline $\begin{array}{l}\text { Boinon et al., } 2014 \\
\text { (France) }\end{array}$ & Longitudinal & $\begin{array}{l}102 \text { women } \\
\text { with breast } \\
\text { cancer }\end{array}$ & French (100\%) & $\begin{array}{l}52.9 \\
(S D=10.2)\end{array}$ & $\begin{array}{l}\text { Surgery }(31.4 \%) \\
C T x+R T x(100 \%)\end{array}$ \\
\hline $\begin{array}{l}\text { Hasson-Ohayon et } \\
\text { al., } 2014 \text { (Israel) }\end{array}$ & $\begin{array}{l}\text { Secondary } \\
\text { analysis }\end{array}$ & $\begin{array}{l}150 \text { women } \\
\text { with } \\
\text { advanced } \\
\text { breast cancer }\end{array}$ & Israel (100\%) & $\begin{array}{l}\text { Younger: } \\
45.67 \\
(S D=6.55) \\
\text { Older: } \\
62.16 \\
(S D=5.70)\end{array}$ & NR \\
\hline $\begin{array}{l}\text { Hughes et al., } 2014 \\
\text { (USA) }\end{array}$ & Longitudinal & $\begin{array}{l}164 \text { women } \\
\text { with breast } \\
\text { cancer stage } \\
0 \text {-IIIA }\end{array}$ & $\begin{array}{l}\text { White }(80.5 \%) \\
\text { Black }(12.8 \%) \\
\text { Other }(6.7 \%)\end{array}$ & $\begin{array}{l}56.13 \\
(S D=11.47)\end{array}$ & $\begin{array}{l}\text { Surgery }(32.9 \%) ; \\
\text { Surgery+RTX }(27.4 \%) ; \\
\text { Surgery+CTX } \\
(15.2 \%) ; \\
\text { Surgery+RTX+CTX } \\
(23.8 \%)\end{array}$ \\
\hline
\end{tabular}




\begin{tabular}{|c|c|c|c|c|c|}
\hline \multirow{2}{*}{$\begin{array}{l}\text { Author (year), } \\
\text { Country }\end{array}$} & \multirow{2}{*}{$\begin{array}{l}\text { Study } \\
\text { design }\end{array}$} & \multicolumn{4}{|c|}{ Sample characteristics } \\
\hline & & $\mathbf{N}$ & Race/ethnicity & $\begin{array}{l}\text { Age } \\
\text { (mean, SD) }\end{array}$ & Tx \\
\hline $\begin{array}{l}\text { Schleife et al., } 2014 \\
\text { (Germany) }\end{array}$ & $\begin{array}{l}\text { Secondary } \\
\text { analysis }\end{array}$ & $\begin{array}{l}107 \text { women } \\
\text { with breast } \\
\text { cancer }\end{array}$ & Not reported & $\begin{array}{l}56.4 \\
(S D=10.5)\end{array}$ & $\begin{array}{l}\text { Surgery }(96 \%) ; C T x \\
(98 \%)\end{array}$ \\
\hline $\begin{array}{l}\text { Wang et al., } 2014 \\
\text { (China) }\end{array}$ & $\begin{array}{l}\text { Cross- } \\
\text { sectional }\end{array}$ & $\begin{array}{l}123 \text { women } \\
\text { with breast } \\
\text { cancer }\end{array}$ & Chinese (100\%) & $\begin{array}{l}49.7 \\
(S D=9.6)\end{array}$ & NR \\
\hline $\begin{array}{l}\text { Borstelmann et al., } \\
2015 \text { (USA) }\end{array}$ & $\begin{array}{l}\text { Secondary } \\
\text { analysis }\end{array}$ & $\begin{array}{l}675 \text { women } \\
\text { with breast } \\
\text { cancer stage I- } \\
\text { III }\end{array}$ & $\begin{array}{l}\text { White (86\%); Non- } \\
\text { white (14\%) }\end{array}$ & 35.4 & $\begin{array}{l}\text { Surgery (84\%); CTx } \\
(76 \%)\end{array}$ \\
\hline $\begin{array}{l}\text { Ozkaraman et al., } \\
2015 \text { (Turkey) }\end{array}$ & $\begin{array}{l}\text { Cross- } \\
\text { sectional }\end{array}$ & $\begin{array}{l}128 \text { breast } \\
\text { cancer } \\
\text { patients }\end{array}$ & Not reported & $\begin{array}{l}51.13 \\
(\mathrm{SD}=8.48)\end{array}$ & NR \\
\hline $\begin{array}{l}\text { Alfonsson et al., } \\
2016 \text { (Sweden) }\end{array}$ & Longitudinal & $\begin{array}{l}833 \text { women } \\
\text { with breast } \\
\text { cancer }\end{array}$ & Sweden (100\%) & $\begin{array}{l}60.6 \\
(S D=11.6)\end{array}$ & $\begin{array}{l}\text { CTx }(38 \%) \\
\text { Target-drug }(8 \%)\end{array}$ \\
\hline $\begin{array}{l}\text { Malicka et al., } 2016 \\
\text { (Poland) }\end{array}$ & $\begin{array}{l}\text { Cross- } \\
\text { sectional }\end{array}$ & $\begin{array}{l}25 \text { women } \\
\text { with breast } \\
\text { cancer }\end{array}$ & Polish (100\%) & $\begin{array}{l}63.2 \\
(\mathrm{SD}=7.0)\end{array}$ & Surgery (100\%) \\
\hline $\begin{array}{l}\text { Berhili et al., } 2017 \\
\text { (Morocco) }\end{array}$ & $\begin{array}{l}\text { Cross- } \\
\text { sectional }\end{array}$ & $\begin{array}{l}446 \text { women } \\
\text { with breast } \\
\text { cancer }\end{array}$ & Not reported & $\begin{array}{l}50 \\
(S D=8)\end{array}$ & $\begin{array}{l}\text { Surgery (21\%); CTx } \\
(38 \%) \\
\text { RTx }(17 \%) ; \text { Hormone } \\
(33 \%)\end{array}$ \\
\hline $\begin{array}{l}\text { Fong et al., } 2017 \\
\text { (Canada) }\end{array}$ & $\begin{array}{l}\text { Secondary } \\
\text { analysis }\end{array}$ & $\begin{array}{l}157 \text { women } \\
\text { with breast } \\
\text { cancer }\end{array}$ & White (85\%) & $\begin{array}{l}55 \\
(S D=11)\end{array}$ & $\begin{array}{l}\text { Lumpectomy } \\
(60.1 \%) ; \\
\text { Mastectomy } \\
(57.8 \%) ; \\
\text { CTx }(63.6 \%) ; R T x \\
(85 \%) ; \\
\text { Hormone }(52.6 \%)\end{array}$ \\
\hline $\begin{array}{l}\text { Moon et al., } 2017 \\
\text { (USA) }\end{array}$ & $\begin{array}{l}\text { Secondary } \\
\text { analysis }\end{array}$ & $\begin{array}{l}661 \text { women } \\
\text { with newly } \\
\text { diagnosed } \\
\text { with breast } \\
\text { cancer }\end{array}$ & $\begin{array}{l}\text { Caucasian (89\%); } \\
\text { Minority }(9.8 \%) ; \text { Not } \\
\text { applicable (1.3\%) }\end{array}$ & $\begin{array}{l}51.18 \\
(S D=9.05)\end{array}$ & NR \\
\hline $\begin{array}{l}\text { Schellekens et al., } \\
2017 \text { (Canada) }\end{array}$ & $\begin{array}{l}\text { Randomized } \\
\text { controlled } \\
\text { Trial }\end{array}$ & $\begin{array}{l}139 \text { women } \\
\text { with breast } \\
\text { cancer stage I- } \\
\text { III } \\
\text { (MBCR; } n=69 \\
\text { and SET; } \\
\mathrm{n}=70 \text { ) }\end{array}$ & Canadian (100\%) & $\begin{array}{l}\text { MBCR: } \\
54.9 \\
(S D=9.2) \\
\text { SET: } \\
53.2 \\
(S D=9.8)\end{array}$ & NR \\
\hline
\end{tabular}




\begin{tabular}{|c|c|c|c|c|c|}
\hline \multirow{2}{*}{$\begin{array}{l}\text { Author (year), } \\
\text { Country }\end{array}$} & \multirow{2}{*}{$\begin{array}{l}\text { Study } \\
\text { design }\end{array}$} & \multicolumn{4}{|c|}{ Sample characteristics } \\
\hline & & $\mathbf{N}$ & Race/ethnicity & $\begin{array}{l}\text { Age } \\
\text { (mean, SD) }\end{array}$ & Tx \\
\hline $\begin{array}{l}\text { Su et al., } 2017 \\
\text { (Taiwan) }\end{array}$ & $\begin{array}{l}\text { Cross- } \\
\text { sectional }\end{array}$ & $\begin{array}{l}300 \text { women } \\
\text { with breast } \\
\text { cancer }\end{array}$ & Taiwanese (100\%) & $\begin{array}{l}48.16 \\
(S D=9.07)\end{array}$ & $\begin{array}{l}R T x(58.7 \%) ; C T X \\
(71.7 \%) \\
\text { Hormone }(70 \%) ; \\
\text { Target-drug }(22.7 \%)\end{array}$ \\
\hline $\begin{array}{l}\text { Thompson et al., } \\
2017 \text { (USA) }\end{array}$ & $\begin{array}{l}\text { Secondary } \\
\text { analysis }\end{array}$ & $\begin{array}{l}227 \text { African } \\
\text { American } \\
\text { women with } \\
\text { breast cancer }\end{array}$ & $\begin{array}{l}\text { African American } \\
(100 \%)\end{array}$ & $\begin{array}{l}56 \\
(S D=10)\end{array}$ & $\begin{array}{l}\text { Surgery }(68.8 \%) ; \\
\operatorname{CTx}(49.6 \%) \\
\text { RTX }(77.4 \%) ; \\
\text { Hormone }(63.1 \%)\end{array}$ \\
\hline $\begin{array}{l}\text { Tomita et al., } 2017 \\
\text { (Japan) }\end{array}$ & $\begin{array}{l}\text { Secondary } \\
\text { analysis }\end{array}$ & $\begin{array}{l}157 \text { women } \\
\text { with breast } \\
\text { cancer }\end{array}$ & Japanese (100\%) & $\begin{array}{l}59.08 \\
(S D=10.06)\end{array}$ & $\begin{array}{l}\text { Surgery (94.9\%);CTX } \\
(50.3 \%) ; \\
\text { RTX } \\
(65.6 \%) ; \text { Hormone } \\
(75.8 \%)\end{array}$ \\
\hline $\begin{array}{l}\text { Bright \& Stanton, } \\
2018 \text { (USA) }\end{array}$ & Longitudinal & $\begin{array}{l}130 \text { women } \\
\text { with breast } \\
\text { cancer }\end{array}$ & $\begin{array}{l}\text { White }(73.1 \%) ; \\
\text { Asian }(9.2 \%) ; \\
\text { Latina (8.5\%); } \\
\text { African American } \\
\text { (3.1\%); Native } \\
\text { American/Alaskan } \\
\text { Native (0.8\%); Other } \\
\text { (5.4\%) }\end{array}$ & $\begin{array}{l}54.2 \\
(S D=11.7)\end{array}$ & $\begin{array}{l}\text { Surgery }(99.2 \%) \\
\text { Hormone }(92.3 \%)\end{array}$ \\
\hline $\begin{array}{l}\text { Schmidt et al., } 2018 \\
\text { (Germany) }\end{array}$ & $\begin{array}{l}\text { Secondary } \\
\text { analysis }\end{array}$ & $\begin{array}{l}225 \text { women } \\
\text { with breast } \\
\text { cancer }\end{array}$ & Germany (100\%) & $\begin{array}{l}54.3 \\
(S D=9.5)\end{array}$ & $\operatorname{CTx}(37.6 \%)$ \\
\hline $\begin{array}{l}\text { Escalera et al., } \\
2019 \text { (USA) }\end{array}$ & $\begin{array}{l}\text { Secondary } \\
\text { analysis }\end{array}$ & $\begin{array}{l}151 \text { Latinas } \\
\text { with breast } \\
\text { cancer stage } \\
0 \text {-Illc }\end{array}$ & Not reported & $\begin{array}{l}50.5 \\
(S D=10.9)\end{array}$ & $\begin{array}{l}\text { Surgery }(100 \%) ; C T x \\
(16.6 \%) ; R T x \\
(27.8 \%) ; C T x+R T x \\
(39.7 \%)\end{array}$ \\
\hline $\begin{array}{l}\text { Wondimagegnehu } \\
\text { et al., 2019 } \\
\text { (Ethiopia) }\end{array}$ & $\begin{array}{l}\text { Cross- } \\
\text { sectional }\end{array}$ & $\begin{array}{l}428 \text { women } \\
\text { with breast } \\
\text { cancer }\end{array}$ & Ethiopian (100\%) & Median $=40$ & NR \\
\hline $\begin{array}{l}\text { Janowski et al., } \\
2020 \text { (Poland) }\end{array}$ & $\begin{array}{l}\text { Cross- } \\
\text { sectional }\end{array}$ & $\begin{array}{l}70 \text { women } \\
\text { with breast } \\
\text { cancer }\end{array}$ & Polish (100\%) & $\begin{array}{l}56.52 \\
(S D=14.18)\end{array}$ & Surgery $(100 \%)$ \\
\hline $\begin{array}{l}\text { Schmidt \& } \\
\text { Andrykowski } \\
2004 \text { (USA) }\end{array}$ & $\begin{array}{l}\text { Cross- } \\
\text { sectional }\end{array}$ & $\begin{array}{l}210 \text { women } \\
\text { with breast } \\
\text { cancer }\end{array}$ & $\begin{array}{l}\text { Caucasian (91\%); } \\
\text { African-American } \\
(1.4 \%) ; \text { Asian (1\%); } \\
\text { Latino/Hispanic } \\
(1 \%) ; \text { Native } \\
\text { American }(0.5 \%) ; \\
\text { Other }(4.3 \%)\end{array}$ & $\begin{array}{l}47.4 \\
(S D=8.4)\end{array}$ & $\begin{array}{l}\text { Surgery }(88.1 \%) ; C T x \\
(26.2 \%) ; R T x \\
(12.9 \%) ; C T x+R T x \\
(52.9 \%)\end{array}$ \\
\hline $\begin{array}{l}\text { Wong et al., } 2018 \\
\text { (USA) }\end{array}$ & $\begin{array}{l}\text { Cross- } \\
\text { sectional }\end{array}$ & $\begin{array}{l}96 \text { Chinese } \\
\text { American } \\
\text { breast cancer } \\
\text { survivors }\end{array}$ & $\begin{array}{l}\text { Chinese-American } \\
(100 \%)\end{array}$ & $\begin{array}{l}54.54 \\
(S D=7.91)\end{array}$ & NR \\
\hline
\end{tabular}




\begin{tabular}{|c|c|c|c|c|c|}
\hline \multirow{2}{*}{$\begin{array}{l}\text { Author (year), } \\
\text { Country }\end{array}$} & \multirow{2}{*}{$\begin{array}{l}\text { Study } \\
\text { design }\end{array}$} & \multicolumn{4}{|c|}{ Sample characteristics } \\
\hline & & $\mathbf{N}$ & Race/ethnicity & $\begin{array}{l}\text { Age } \\
\text { (mean, SD) }\end{array}$ & Tx \\
\hline $\begin{array}{l}\text { Lally et al., } 2019 \\
\text { (USA) }\end{array}$ & RCT & $\begin{array}{l}100 \text { women } \\
\text { within } 0-2 \\
\text { months of } \\
\text { first, stage 0-II } \\
\text { breast cancer } \\
\text { survivors }\end{array}$ & $\begin{array}{l}\text { Caucasian (93\%); } \\
\text { African-American } \\
\text { (3\%); American- } \\
\text { Indian (1\%); Asian } \\
(1 \%)\end{array}$ & $\begin{array}{l}54.2 \\
(S D=9.9)\end{array}$ & NR \\
\hline $\begin{array}{l}\text { Lueboonthavatchai, } \\
2007 \text { (Thailand) }\end{array}$ & $\begin{array}{l}\text { Cross- } \\
\text { sectional }\end{array}$ & $\begin{array}{l}300 \text { women } \\
\text { with breast } \\
\text { cancer }\end{array}$ & Not reported & $\begin{array}{l}\text { Not } \\
\text { reported }\end{array}$ & $\begin{array}{l}50.09 \\
(S D=11.01)\end{array}$ \\
\hline $\begin{array}{l}\text { Mantani et al., } 2007 \\
\text { (Japan) }\end{array}$ & $\begin{array}{l}\text { Cross- } \\
\text { sectional }\end{array}$ & $\begin{array}{l}46 \text { women } \\
\text { with breast } \\
\text { cancer stage I } \\
\text { or II and their } \\
\text { husbands }\end{array}$ & Japanese (100\%) & $\begin{array}{l}\text { Patients: } \\
52.3 \\
(\mathrm{SD}=10.5)\end{array}$ & $\begin{array}{l}\text { Surgery }(100 \%) \\
\text { CTX,RTX, hormone } \\
(87 \%)\end{array}$ \\
\hline $\begin{array}{l}\text { Ashing-Giwa et al., } \\
2013 \text { (USA) }\end{array}$ & $\begin{array}{l}\text { Secondary } \\
\text { analysis }\end{array}$ & $\begin{array}{l}232 \text { women } \\
\text { with Latina } \\
\text { breast cancer } \\
\text { stage } 0 \text {-III }\end{array}$ & $\begin{array}{l}\text { Mexican (73\%); } \\
\text { Central-American } \\
\text { (13\%); South- } \\
\text { American (9\%); US- } \\
\text { born Latinas (5\%) }\end{array}$ & $\begin{array}{l}53 \\
(S D=10.6)\end{array}$ & $\begin{array}{l}\text { Surgery }(95 \%) ; \\
\text { CTx(70\%); RTX } \\
(70 \%) ; \text { Hormone } \\
(66 \%)\end{array}$ \\
\hline $\begin{array}{l}\text { Segrin et al., } 2018 \\
\text { (USA) }\end{array}$ & $\begin{array}{l}\text { Cross- } \\
\text { sectional }\end{array}$ & $\begin{array}{l}230 \text { dyads of } \\
\text { Latinas with } \\
\text { breast cancer } \\
\text { and their } \\
\text { family } \\
\text { caregivers }\end{array}$ & $\begin{array}{l}\text { White }(85 \%) ; \\
\text { Hispanic (14\%); } \\
\text { Other (1\%) }\end{array}$ & $\begin{array}{l}\text { Patients: } \\
50.19 \\
(S D=10.4) \\
\text { Caregivers: } \\
44.20 \\
(S D=13.2)\end{array}$ & $\begin{array}{l}\text { Surgery (60\%); CTx } \\
(82.6 \%) \\
\text { RTx }(27 \%) ; \text { Hormone } \\
(14.8 \%)\end{array}$ \\
\hline $\begin{array}{l}\text { Aguado Loi et al., } \\
2013 \text { (USA) }\end{array}$ & $\begin{array}{l}\text { Secondary } \\
\text { analysis }\end{array}$ & $\begin{array}{l}68 \text { Latinas } \\
\text { diagnosed } \\
\text { with breast } \\
\text { cancer }\end{array}$ & $\begin{array}{l}\text { Latino/Hispanic } \\
(100 \%) \text {; }\end{array}$ & $\begin{array}{l}55.4 \\
(S D=10.4)\end{array}$ & $\begin{array}{l}\text { Surgery }(95.6 \%) ; C T x \\
(63.2 \%) ; R T x \\
(48.5 \%) ; \\
\text { Hormone }(69.1 \%)\end{array}$ \\
\hline $\begin{array}{l}\text { Giese-Davis \& } \\
\text { Hermanson, } \\
2000 \text { (USA) }\end{array}$ & $\begin{array}{l}\text { Cross- } \\
\text { sectional }\end{array}$ & $\begin{array}{l}125 \text { women } \\
\text { with } \\
\text { metastatic } \\
\text { breast cancer }\end{array}$ & $\begin{array}{l}\text { Caucasian (87\%); } \\
\text { Asian-American } \\
(6 \%) ; \text { Hispanic- } \\
\text { Latina (2\%); Native } \\
\text { American (2\%); } \\
\text { African-American } \\
\text { (1\%); Other (2\%) }\end{array}$ & $\begin{array}{l}53 \\
(S D=10.7)\end{array}$ & NR \\
\hline $\begin{array}{l}\text { Manne et al., } 2007 \\
\text { (USA) }\end{array}$ & $\begin{array}{l}\text { Secondary } \\
\text { analysis }\end{array}$ & $\begin{array}{l}235 \text { women } \\
\text { with breast } \\
\text { cancer and } \\
\text { their } \\
\text { significant } \\
\text { others }\end{array}$ & $\begin{array}{l}\text { Caucasian } \\
\text { (patients: } 89 \% \text { and } \\
\text { partners: } 91 \% \text { ) }\end{array}$ & $\begin{array}{l}50 \\
(\mathrm{SD}=9.9)\end{array}$ & $\begin{array}{l}\text { Surgery (100\%); CTx } \\
(75 \%) ; R T x(13 \%)\end{array}$ \\
\hline
\end{tabular}




\begin{tabular}{|c|c|c|c|c|c|}
\hline \multirow{2}{*}{$\begin{array}{l}\text { Author (year), } \\
\text { Country }\end{array}$} & \multirow{2}{*}{$\begin{array}{l}\text { Study } \\
\text { design }\end{array}$} & \multicolumn{4}{|c|}{ Sample characteristics } \\
\hline & & $\mathbf{N}$ & Race/ethnicity & $\begin{array}{l}\text { Age } \\
\text { (mean, SD) }\end{array}$ & Tx \\
\hline $\begin{array}{l}\text { Segrin et al., } 2007 \\
\text { (USA) }\end{array}$ & $\begin{array}{l}\text { Secondary } \\
\text { analysis }\end{array}$ & $\begin{array}{l}96 \text { dyads of } \\
\text { women with } \\
\text { breast cancer } \\
\text { stage I-III and } \\
\text { their partners }\end{array}$ & $\begin{array}{l}\text { White }(85 \%) ; \\
\text { Hispanic (14\%); } \\
\text { Other (1\%) }\end{array}$ & $\begin{array}{l}\text { Patients: } \\
54.11 \\
\text { (SD=10.6) } \\
\text { Partners: } \\
51.68 \\
(S D=14.8)\end{array}$ & $\begin{array}{l}\text { CTx }(75 \%) ; R T x \\
(54 \%) ; \text { Hormone } \\
(36 \%)\end{array}$ \\
\hline $\begin{array}{l}\text { Al-Zaben et al., } \\
2015 \text { (Saudi } \\
\text { Arabia) }\end{array}$ & $\begin{array}{l}\text { Cross- } \\
\text { sectional }\end{array}$ & $\begin{array}{l}49 \text { married } \\
\text { women with } \\
\text { breast cancer }\end{array}$ & Arabs (100\%) & $\begin{array}{l}48.9 \\
(S D=7.1)\end{array}$ & $\begin{array}{l}\text { Surgery (89.8\%); } \\
\text { CTx(83.7\%); RTx } \\
(57.1 \%)\end{array}$ \\
\hline $\begin{array}{l}\text { Simpson et al., } \\
2002 \text { (Canada) }\end{array}$ & RCT & $\begin{array}{l}89 \text { women } \\
\text { with breast } \\
\text { cancer }\end{array}$ & Not reported & $\begin{array}{l}49.3 \\
(S D=7.7)\end{array}$ & Not reported \\
\hline $\begin{array}{l}\text { Brothers \& } \\
\text { Andersen, } 2009 \\
\text { (USA) }\end{array}$ & Longitudinal & $\begin{array}{l}67 \text { women } \\
\text { with breast } \\
\text { cancer }\end{array}$ & $\begin{array}{l}\text { Caucasian }(93 \%) ; \\
\text { African-American } \\
(7 \%)\end{array}$ & $\begin{array}{l}54 \\
(S D=11)\end{array}$ & $\begin{array}{l}\text { Surgery }(28 \%) ; C T X \\
(43 \%) ; \text { RTX }(19 \%) ; \\
\text { Hormone }(39 \%)\end{array}$ \\
\hline $\begin{array}{l}\text { Gagliardi et al., } \\
2009 \text { (Italy) }\end{array}$ & $\begin{array}{l}\text { Cross- } \\
\text { sectional }\end{array}$ & $\begin{array}{l}47 \text { women } \\
\text { with breast } \\
\text { cancer at low } \\
\text { or } \\
\text { intermediate } \\
\text { high risk }\end{array}$ & Italian (100\%) & $\begin{array}{l}54.28 \\
(S D=8.4)\end{array}$ & Surgery $(100 \%)$ \\
\hline $\begin{array}{l}\text { Puigpinos-Riera et } \\
\text { al., } 2018 \text { (Spain) }\end{array}$ & $\begin{array}{l}\text { Secondary } \\
\text { analysis }\end{array}$ & $\begin{array}{l}2235 \text { women } \\
\text { with breast } \\
\text { cancer }\end{array}$ & Spanish (100\%) & NR & NR \\
\hline $\begin{array}{l}\text { Wang et al., } 2019 \\
\text { (USA) }\end{array}$ & $\begin{array}{l}\text { Cross- } \\
\text { sectional }\end{array}$ & $\begin{array}{l}436 \text { breast } \\
\text { cancer } \\
\text { patients with } \\
\text { stage } 0 \text {-III }\end{array}$ & Chinese (100\%) & $\begin{array}{l}21-50 \text { yrs } \\
(27.52 \%), \\
51-64 \text { yrs } \\
(48.17 \%), \\
65 \text { or older } \\
\text { yrs } \\
(24.31 \%)\end{array}$ & NR \\
\hline
\end{tabular}

\subsection{Association between social relationships and affective symptoms}

In this review, social relationships were classified as functional and structural aspects of social relations. Of the included 65 studies, 60 focused on functional aspects of social relationships, and the remaining 5 reported on structural aspects of social relationships. Interestingly, none of the included 65 studies examined the association between social relationships and cognitive symptoms of breast cancer patients; thus, in this paper, we focused only on the affective symptoms of breast cancer patients and their association with patients' social relationships. 
Table 3

Characteristics of studies regarding social relationships associated with the patient's affective symptoms

\begin{tabular}{|c|c|c|c|c|}
\hline $\begin{array}{l}\text { Author (year), } \\
\text { Country }\end{array}$ & $\begin{array}{l}\text { Social relationship } \\
\text { (measures) }\end{array}$ & $\begin{array}{l}\text { Affective } \\
\text { symptoms } \\
\text { (measures) }\end{array}$ & Analysis adjust for & Key findings \\
\hline \multicolumn{5}{|c|}{ Functional Aspect of Social Relationships } \\
\hline \multicolumn{5}{|l|}{ Social support } \\
\hline \multirow[t]{2}{*}{$\begin{array}{l}\text { Roberts et al.,1994 } \\
\text { (USA) }\end{array}$} & Social support (SSQ) & $\begin{array}{l}\text { Psychological } \\
\text { status (SCL- } \\
90-R) \& \\
\text { (distress GSI) }\end{array}$ & Desirability & $\begin{array}{l}\text { 1) Single patients who } \\
\text { had support from friend } \\
\text { demonstrated lower } \\
\text { depression }(r=-0.44) \text {, } \\
\text { anxiety }(r=-0.38) \text {, and } \\
\text { overall severity of } \\
\text { psychological distress } \\
(r=-0.41) \text { (all } p<0.05) \text {. }\end{array}$ \\
\hline & & & & $\begin{array}{l}\text { 2) Married patients who } \\
\text { had support from } \\
\text { spouse demonstrated } \\
\text { lower depression } \\
\text { (r=-0.27) as well as } \\
\text { overall severity of } \\
\text { psychological distress } \\
(r=-0.27) \text { (both } p<0.01) \text {. }\end{array}$ \\
\hline
\end{tabular}




\begin{tabular}{|c|c|c|c|c|}
\hline $\begin{array}{l}\text { Author (year), } \\
\text { Country }\end{array}$ & $\begin{array}{l}\text { Social relationship } \\
\text { (measures) }\end{array}$ & $\begin{array}{l}\text { Affective } \\
\text { symptoms } \\
\text { (measures) }\end{array}$ & Analysis adjust for & Key findings \\
\hline $\begin{array}{l}\text { Neuling et al., } 1988 \\
\text { (Australia) }\end{array}$ & $\begin{array}{l}\text { Social support } \\
\text { (MDSS) }\end{array}$ & $\begin{array}{l}\text { Anxiety } \\
\text { (STAI) } \\
\text { Depression } \\
\text { (Wakefield } \\
\text { Self- } \\
\text { Assessment } \\
\text { Depression } \\
\text { Inventory) }\end{array}$ & Not reported & $\begin{array}{l}\text { In hospital, } \\
\text { 1) Anxiety was related to } \\
\text { amount of support } \\
\text { received from friends } \\
{[F(1,49)=5.84 ; p<0.05]} \\
\text { and satisfaction with } \\
\text { support from family } \\
\text { members }[F(1,50)=4.54 \text {, } \\
\text { p<0.05]. } \\
\text { 2) Depression was } \\
\text { related to the amount of } \\
\text { support from friends } \\
{[F(1,49)=6.50, p<0.05]} \\
\text { and satisfaction with } \\
\text { family support } \\
{[F(1,50)=5.58, p<0.05] \text {. }} \\
\text { 1-month post operation, } \\
\text { Anxiety was related to } \\
\text { the amount of support } \\
\text { received from friends } \\
{[F(1,37)=6.77, p<0.05)} \\
\text { 3-month post operation, } \\
\text { 1) Anxiety was not } \\
\text { related to the amount of } \\
\text { support but was related } \\
\text { to satisfaction with } \\
\text { support from family } \\
{[F(1,34)=9.72, p<0.005]} \\
\text { 2) Depression was } \\
\text { related to satisfaction } \\
\text { with support from } \\
\text { family }[F(1,34)=5.60, \\
p<0.05]\end{array}$ \\
\hline $\begin{array}{l}\text { Koopman et al., } \\
1998 \text { (USA) }\end{array}$ & $\begin{array}{l}\text { Social support } \\
\text { (Yale Social Support } \\
\text { Index \& single item } \\
\text { measure) }\end{array}$ & $\begin{array}{l}\text { Mood } \\
\text { disturbance } \\
\text { (POMS) }\end{array}$ & Not reported & $\begin{array}{l}\text { Patients' mood } \\
\text { disturbances were } \\
\text { positively associated } \\
\text { with aversive social } \\
\text { support. }\end{array}$ \\
\hline $\begin{array}{l}\text { Lee et al., 2004, } \\
\text { (Korea) }\end{array}$ & Social Support (SSS) & $\begin{array}{l}\text { Mood } \\
\text { disturbance } \\
\text { (Linear } \\
\text { Analogue Self- } \\
\text { Assessment } \\
\text { Scale) }\end{array}$ & Not reported & $\begin{array}{l}\text { Patients with low social } \\
\text { support reported higher } \\
\text { mood disturbance } \\
(r=-0.25, p=0.004)\end{array}$ \\
\hline
\end{tabular}




\begin{tabular}{|c|c|c|c|c|}
\hline $\begin{array}{l}\text { Author (year), } \\
\text { Country }\end{array}$ & $\begin{array}{l}\text { Social relationship } \\
\text { (measures) }\end{array}$ & $\begin{array}{l}\text { Affective } \\
\text { symptoms } \\
\text { (measures) }\end{array}$ & Analysis adjust for & Key findings \\
\hline $\begin{array}{l}\text { Maly et al., 2005, } \\
\text { (USA) }\end{array}$ & $\begin{array}{l}\text { Emotional } \\
\text { \&lnstrumental support } \\
\text { (items developed based } \\
\text { on qualitative } \\
\text { interview) }\end{array}$ & $\begin{array}{l}\text { Depression } \\
\text { (CES-D) } \\
\text { Anxiety (STAl- } \\
\text { S) }\end{array}$ & $\begin{array}{l}\text { Socio- } \\
\text { demographics, } \\
\text { cancer stage, } \\
\text { treatment type, } \\
\text { comorbidity }\end{array}$ & $\begin{array}{l}\text { 1) Patients } \\
\text { demonstrated lower } \\
\text { depressive when they } \\
\text { had partners who helped } \\
\text { around the house ( } \beta \\
=-0.16, p=0.048) \text {. } \\
\text { 2) In white women } \\
\text { (patients), they showed } \\
\text { more anxiety when they } \\
\text { had other family } \\
\text { members or friends who } \\
\text { helped with bathing or } \\
\text { dressing ( } \beta=0.20 \text {, } \\
p=0.028) \\
\text { 3) In non-white women } \\
\text { (patients), their anxiety } \\
\text { became lower when they } \\
\text { had children who } \\
\text { listened to concerns or } \\
\text { worries ( } \beta=-0.30 \text {, } \\
p=0.044) \text { and helped } \\
\text { around the house ( } \beta \\
=-0.25, p=0.046 \text { ). } \\
\text { 4) In non-white women } \\
\text { (patients), their } \\
\text { depression became } \\
\text { lower when they had } \\
\text { children who helped } \\
\text { around the house ( } \beta \\
=-0.30, p=0.02 \text { ). }\end{array}$ \\
\hline $\begin{array}{l}\text { Palesh et al., 2006, } \\
\text { (USA) }\end{array}$ & $\begin{array}{l}\text { Social support (UCLA } \\
\text { Social Support } \\
\text { Inventory) }\end{array}$ & $\begin{array}{l}\text { Mood } \\
\text { disturbance } \\
\text { (POMS) }\end{array}$ & Not reported & $\begin{array}{l}\text { No relationship was } \\
\text { found between mood } \\
\text { disturbances and } \\
\text { satisfaction with social } \\
\text { support }\end{array}$ \\
\hline $\begin{array}{l}\text { Porter et al., 2006, } \\
\text { (USA) }\end{array}$ & $\begin{array}{l}\text { Social support } \\
\text { satisfaction (SSQ) }\end{array}$ & $\begin{array}{l}\text { Negative } \\
\text { mood (POMS- } \\
\text { SF) }\end{array}$ & Not reported & $\begin{array}{l}\text { Patients demonstrated } \\
\text { less negative mood } \\
\text { state when their } \\
\text { satisfaction with social } \\
\text { support increased ( } \beta \\
=-0.087, t=-2.041) .\end{array}$ \\
\hline $\begin{array}{l}\text { Friedman et al., } \\
\text { 2006, (USA) }\end{array}$ & Social support (SSQ) & $\begin{array}{l}\text { Mood } \\
\text { disturbances } \\
\text { (TMD and } \\
\text { POMS-SF) }\end{array}$ & Not reported & $\begin{array}{l}\text { No association was } \\
\text { found between mood } \\
\text { disturbances and social } \\
\text { support }\end{array}$ \\
\hline $\begin{array}{l}\text { Kim \& Morrow, } \\
2007 \text { (USA) }\end{array}$ & Family support (FES) & $\begin{array}{l}\text { Anxiety } \\
\text { (STAl) }\end{array}$ & Emetic score & $\begin{array}{l}\text { Higher family support } \\
\text { predicted lower patients' } \\
\text { anxiety level ( } \beta=-0.36 \text {, } \\
p<0.001) \text {. }\end{array}$ \\
\hline $\begin{array}{l}\text { Nausheen \& Kamal, } \\
2007 \text { (Pakistan) }\end{array}$ & $\begin{array}{l}\text { Familial social support } \\
\text { (FSSS) }\end{array}$ & $\begin{array}{l}\text { Depression } \\
\text { (SSDS) }\end{array}$ & Not reported & $\begin{array}{l}\text { 1) Patients showed less } \\
\text { depression when they } \\
\text { had strong familiar } \\
\text { support ( } r=-0.85 \text {, } \\
p<0.001)\end{array}$ \\
\hline
\end{tabular}




\begin{tabular}{|c|c|c|c|c|}
\hline $\begin{array}{l}\text { Author (year), } \\
\text { Country }\end{array}$ & $\begin{array}{l}\text { Social relationship } \\
\text { (measures) }\end{array}$ & $\begin{array}{l}\text { Affective } \\
\text { symptoms } \\
\text { (measures) }\end{array}$ & Analysis adjust for & Key findings \\
\hline \multirow[t]{5}{*}{$\begin{array}{l}\text { Von Ah \& Kang, } \\
2008 \text { (USA) }\end{array}$} & \multirow[t]{5}{*}{$\begin{array}{l}\text { Emotional and aid } \\
\text { support (NSSQ) }\end{array}$} & \multirow[t]{5}{*}{$\begin{array}{l}\text { Mood } \\
\text { disturbance } \\
\text { (POMS-SF) }\end{array}$} & \multirow[t]{5}{*}{ Not reported } & $\begin{array}{l}\text { 1) Emotional support } \\
\text { was associated with } \\
\text { mood disturbance } \\
\text { before }(r=-.34, p<.01) \text {, } \\
\text { after }(r=-.47, p<.001) \\
\text { adjuvant therapy. }\end{array}$ \\
\hline & & & & $\begin{array}{l}\text { 2) Aid support was } \\
\text { associated with mood } \\
\text { disturbances during } \\
(r=-.38, p<.001) \text { adjuvan } \\
\text { therapy. }\end{array}$ \\
\hline & & & & $\begin{array}{l}\text { 3) Prior to adjuvant } \\
\text { therapy, aid support had } \\
\text { indirect effect on mood } \\
\text { disturbance whereas } \\
\text { emotional support has } \\
\text { both indirect ( } B=-0.42 \text {, } \\
p<.05) \text { and direct effect } \\
\text { ( }(=-0.53) \text { on mood } \\
\text { disturbances }\end{array}$ \\
\hline & & & & $\begin{array}{l}\text { 4) During adjuvant } \\
\text { therapy, aid support has } \\
\text { indirect effect of mood } \\
\text { disturbance }\end{array}$ \\
\hline & & & & $\begin{array}{l}\text { 5) After adjuvant } \\
\text { therapy, emotional } \\
\text { support has both direct } \\
\text { ( }(=-0.39, p<0.01) \text { and } \\
\text { indirect effects on mooc } \\
\text { disturbances. }\end{array}$ \\
\hline $\begin{array}{l}\text { Gellaitry et al., } \\
\text { 2010, (UK) }\end{array}$ & $\begin{array}{l}\text { Social Support } \\
\text { (Significant Others } \\
\text { Scale) }\end{array}$ & $\begin{array}{l}\text { Psychological } \\
\text { well-being } \\
\text { (POMS) }\end{array}$ & Baseline measures & $\begin{array}{l}\text { In intervention group, } \\
\text { patients demonstrated } \\
\text { less depression when } \\
\text { they were satisfied with } \\
\text { emotional support } \\
(p<0.05)\end{array}$ \\
\hline \multirow{2}{*}{$\begin{array}{l}\text { Gorman et al., } \\
\text { 2010, (USA) }\end{array}$} & Social support & \multirow{2}{*}{$\begin{array}{l}\text { Depressive } \\
\text { symptoms }\end{array}$} & \multirow{2}{*}{$\begin{array}{l}\text { Demographic and } \\
\text { clinical } \\
\text { characteristics, } \\
\text { randomized } \\
\text { assignment }\end{array}$} & \multirow{2}{*}{$\begin{array}{l}\text { Patients with greater } \\
\text { social support showed } \\
\text { lower depressive } \\
\text { symptoms }(p<0.0001)\end{array}$} \\
\hline & (MOS-SSS) & & & \\
\hline
\end{tabular}




\begin{tabular}{|c|c|c|c|c|}
\hline $\begin{array}{l}\text { Author (year), } \\
\text { Country }\end{array}$ & $\begin{array}{l}\text { Social relationship } \\
\text { (measures) }\end{array}$ & $\begin{array}{l}\text { Affective } \\
\text { symptoms } \\
\text { (measures) }\end{array}$ & Analysis adjust for & Key findings \\
\hline \multirow[t]{4}{*}{$\begin{array}{l}\text { Hasson-Ohayon et } \\
\text { al., 2010, (Israel) }\end{array}$} & \multirow[t]{4}{*}{$\begin{array}{l}\text { Agent of Support and } \\
\text { Type of Support } \\
\text { (CPASS) }\end{array}$} & \multirow[t]{4}{*}{$\begin{array}{l}\text { Psychological } \\
\text { distress (BSI) }\end{array}$} & \multirow[t]{4}{*}{ Not reported } & $\begin{array}{l}\text { 1) Patients } \\
\text { demonstrated lower } \\
\text { depression when they } \\
\text { had support from } \\
\text { spouse }(r=-0.16, p<0.05) \text {, } \\
\text { family }(r=-0.28, p<0.01) \text {, } \\
\text { and friends }(r=-0.24 \text {, } \\
p<0.01) \text {. }\end{array}$ \\
\hline & & & & $\begin{array}{l}20 \text { Patients } \\
\text { demonstrated lower } \\
\text { anxiety when they had } \\
\text { support from family } \\
(\mathrm{r}=-0.22, p<0.01)\end{array}$ \\
\hline & & & & $\begin{array}{l}\text { 3) Higher family support } \\
\text { predicted lower patient's } \\
\text { psychological distress } \\
(\beta=-0.32, p<0.005) \text {. }\end{array}$ \\
\hline & & & & $\begin{array}{l}\text { 4) Higher family support } \\
\text { predicted lower } \\
\text { depression and anxiety } \\
(\beta=-0.20, p<0.0057 ; \beta \\
=-0.19, p<0.052) \text {. }\end{array}$ \\
\hline \multirow[t]{2}{*}{$\begin{array}{l}\text { Kim et al., 2010, } \\
\text { (USA) }\end{array}$} & \multirow[t]{2}{*}{$\begin{array}{l}\text { Social support } \\
\text { (developed from } \\
\text { previous studies using } \\
\text { six items) }\end{array}$} & \multirow[t]{2}{*}{$\begin{array}{l}\text { Emotional } \\
\text { well-being } \\
\text { (FACT-B) }\end{array}$} & \multirow[t]{2}{*}{$\begin{array}{l}\text { Age, education } \\
\text { level, race, living } \\
\text { status, cancer } \\
\text { stage }\end{array}$} & $\begin{array}{l}\text { 1) Patients with strong } \\
\text { social support reported } \\
\text { good emotional well- } \\
\text { being }(r=.34, p<.001)\end{array}$ \\
\hline & & & & $\begin{array}{l}\text { 2) Social support } \\
\text { influenced emotional } \\
\text { well-being }(B=0.23 \text {, } \\
p<.001)\end{array}$ \\
\hline $\begin{array}{l}\text { Talley et al., 2010, } \\
\text { (USA) }\end{array}$ & $\begin{array}{l}\text { Partner social support } \\
\text { (Items developed by } \\
\text { Alferi et al, 2001) }\end{array}$ & $\begin{array}{l}\text { Depression } \\
\text { (CES-D) }\end{array}$ & $\begin{array}{l}\text { Age, income co- } \\
\text { morbid illness, co- } \\
\text { residence, distance } \\
\text { from radiation } \\
\text { treatment center, } \\
\text { level of physical } \\
\text { symptoms }\end{array}$ & $\begin{array}{l}\text { Patients showed lower } \\
\text { levels of depression } \\
\text { when they had greater } \\
\text { partner emotional } \\
\text { support }(\beta=-0.23 \text {, } \\
p<0.05) \text {. }\end{array}$ \\
\hline $\begin{array}{l}\text { Cohen et al., 2010, } \\
\text { (Israel) }\end{array}$ & $\begin{array}{l}\text { Perceived social } \\
\text { support } \\
\text { (self-report 10-item) }\end{array}$ & $\begin{array}{l}\text { Emotional } \\
\text { distress (BSI- } \\
18)\end{array}$ & Demographics & $\begin{array}{l}\text { Perceived social support } \\
\text { predicted the variance of } \\
\text { emotional distress ( } \beta \\
=-0.30, p<0.01 \text { ) }\end{array}$ \\
\hline
\end{tabular}




\begin{tabular}{|c|c|c|c|c|}
\hline $\begin{array}{l}\text { Author (year), } \\
\text { Country }\end{array}$ & $\begin{array}{l}\text { Social relationship } \\
\text { (measures) }\end{array}$ & $\begin{array}{l}\text { Affective } \\
\text { symptoms } \\
\text { (measures) }\end{array}$ & Analysis adjust for & Key findings \\
\hline $\begin{array}{l}\text { Hill et al., 2011, } \\
\text { (UK) }\end{array}$ & $\begin{array}{l}\text { Perceived emotional } \\
\text { support (MOS-SSS) }\end{array}$ & $\begin{array}{l}\text { MD (Major } \\
\text { depression) } \\
\text { GAD } \\
\text { (Generalized } \\
\text { anxiety } \\
\text { disorder) }\end{array}$ & $\begin{array}{l}\text { History of } \\
\text { psychiatric } \\
\text { disorder }\end{array}$ & $\begin{array}{l}\text { 1) During one year after } \\
\text { cancer diagnosis, low } \\
\text { social support predicted } \\
\text { onset of MD (OR=2.20, } \\
95 \% \mathrm{Cl}=1.12-4.33, \\
\mathrm{p}<0.05) \text { and } \mathrm{GAD} \\
(\mathrm{OR}=2.51,95 \% \mathrm{Cl}=1.05- \\
5.97, \mathrm{p}<0.03) \\
\text { 2) Low social support } \\
\text { predicted the risk of } \\
\text { onset of both } \mathrm{MD} \\
(\mathrm{OR}=3.43,95 \% \mathrm{Cl}=1.32- \\
8.87, \mathrm{p}=0.01) \text { and } \mathrm{GAD} \\
(\mathrm{OR}=4.00,95 \% \mathrm{Cl}=1.42- \\
11.30, \mathrm{p}=0.01)\end{array}$ \\
\hline $\begin{array}{l}\text { Lee et al., 2011, } \\
\text { (Korea) }\end{array}$ & $\begin{array}{l}\text { Perceived Social } \\
\text { Support (MOS-SSS) }\end{array}$ & $\begin{array}{l}\text { Depressive } \\
\text { Mood (SDS) }\end{array}$ & $\begin{array}{l}\text { Demographics, } \\
\text { menopausal } \\
\text { status, BMI, } \\
\text { exercise, drinking } \\
\text { status. }\end{array}$ & $\begin{array}{l}\text { Worsen emotional } \\
\text { support }(p<0.001) \text {, } \\
\text { informational }(p=0.04) \\
\text { were associated with } \\
\text { deteriorated depressive } \\
\text { mood. }\end{array}$ \\
\hline $\begin{array}{l}\text { Liu et al., 2011, } \\
\text { (China) }\end{array}$ & $\begin{array}{l}\text { Social support (social } \\
\text { support rating scale) }\end{array}$ & $\begin{array}{l}\text { Anxiety and } \\
\text { depression } \\
\text { (HADS) } \\
\text { Psychological } \\
\text { stress } \\
\text { (Psychological } \\
\text { stress scale) }\end{array}$ & Not reported & $\begin{array}{l}\text { 1) Patients' } \\
\text { psychological stress } \\
\text { were associated with } \\
\text { social support } \\
\text { (subjective and } \\
\text { objective) and its utility } \\
\text { (all } p<0.05 \text { ) } \\
\text { 2) Both anxiety } \\
(r=-0.196, p<0.01 ; \\
r=-0.128, p<0.05) \text { and } \\
\text { depression }(r=-0.141, \\
p<0.01 ; r=-0.168, p<0.01) \\
\text { were associated with } \\
\text { objective support and its } \\
\text { utility. } \\
\text { 3) Depression was } \\
\text { associated with } \\
\text { subjective social } \\
\text { support }(r=-0.315, \\
p<0.01) \text {. } \\
\text { 4) Subjective social } \\
\text { support }(\beta=-0.108, \\
p<0.05) \text { and its utility ( } \beta \\
=0.329, p<0.05) \text { were } \\
\text { predictors of anxiety. } \\
\text { 5) Objective social } \\
\text { support }(\beta=-0.249 \text {, } \\
p<0.05) \text { was a predictor } \\
\text { of depression }\end{array}$ \\
\hline
\end{tabular}




\begin{tabular}{|c|c|c|c|c|}
\hline $\begin{array}{l}\text { Author (year), } \\
\text { Country }\end{array}$ & $\begin{array}{l}\text { Social relationship } \\
\text { (measures) }\end{array}$ & $\begin{array}{l}\text { Affective } \\
\text { symptoms } \\
\text { (measures) }\end{array}$ & Analysis adjust for & Key findings \\
\hline $\begin{array}{l}\text { Boinon et al., 2012, } \\
\text { (France) }\end{array}$ & $\begin{array}{l}\text { Perceived social } \\
\text { support (Cancer- } \\
\text { specific questionnaire } \\
\text { of social support) }\end{array}$ & $\begin{array}{l}\text { Depressive } \\
\text { symptom } \\
\text { (BDI-SF) } \\
\text { Negative } \\
\text { affect } \\
\text { (PANAS) }\end{array}$ & $\begin{array}{l}\text { Demographics, } \\
\text { time since surgery, } \\
\text { social sharing } \\
\text { variables }\end{array}$ & $\begin{array}{l}\text { Patients with higher } \\
\text { perceived negative } \\
\text { support demonstrated } \\
\text { higher depressive } \\
\text { symptoms and negative } \\
\text { affect }(B=0.24, p<0.05 \text {; } \\
B=0,26, p<0.01)\end{array}$ \\
\hline $\begin{array}{l}\text { Jones et al., 2012, } \\
\text { (Canada) }\end{array}$ & $\begin{array}{l}\text { Social support } \\
\text { (MSPSS) } \\
\text { Unsupportive social } \\
\text { interactions (USII) }\end{array}$ & $\begin{array}{l}\text { Health anxiety } \\
\text { (MIHT) } \\
\text { Anxiety and } \\
\text { depression } \\
\text { (HADS) }\end{array}$ & $\begin{array}{l}\text { Demographics, } \\
\text { cancer-related } \\
\text { variables, general } \\
\text { anxiety and } \\
\text { depression }\end{array}$ & $\begin{array}{l}\text { 1) Patients had a } \\
\text { tendency to worry about } \\
\text { their health (health } \\
\text { anxiety-affective } \\
\text { dimension) when they } \\
\text { had unsupportive social } \\
\text { interactions ( } r=0.36 \text {, } \\
p<0.001 \text { ) } \\
\text { 2) Patient's health } \\
\text { anxiety-affective } \\
\text { dimension was } \\
\text { predicted by } \\
\text { unsupportive } \\
\text { interactions ( } \beta=0.21 \text {, } \\
p<0.05) \text { and social } \\
\text { support ( } \beta=-0.20 \text {, } \\
p<0.05 \text { ). } \\
\text { 3) Patients reported } \\
\text { higher anxiety when } \\
\text { they had lower perceived } \\
\text { social support }(r=-0.32 \text {, } \\
p<0.001 \text { ) and } \\
\text { unsupportive social } \\
\text { interactions ( } r=0.41 \text {, } \\
p<0.001 \text { ) } \\
\text { 4) Patients reported } \\
\text { higher depression when } \\
\text { they had lower perceived } \\
\text { social support }(r=-0.33 \text {, } \\
p<0.001 \text { ) and } \\
\text { unsupportive social } \\
\text { interactions ( } r=0.44, \\
p<0.001 \text { ) }\end{array}$ \\
\hline $\begin{array}{l}\text { Mallinckordt et al., } \\
2012, \text { (USA) }\end{array}$ & Social support (SPS-M) & $\begin{array}{l}\text { Psychological } \\
\text { distress (BSI) }\end{array}$ & Not reported & $\begin{array}{l}\text { 1) Patients } \\
\text { demonstrated less } \\
\text { psychological distress } \\
\text { when they had higher } \\
\text { social support } \\
(\mathrm{T} 1: \mathrm{r}=-0.26, \mathrm{p}<0.01 ; \mathrm{T} 2 \text { : } \\
\mathrm{r}=-0.44, \mathrm{p}<0.01) \text {. } \\
\text { 2) A significant } \\
\text { association was found } \\
\text { between social support } \\
\text { and psychological } \\
\text { distress at both } \mathrm{T} 1 \text { and } \\
\mathrm{T} 2 \text { (all } p<0.01)\end{array}$ \\
\hline
\end{tabular}




\begin{tabular}{|c|c|c|c|c|}
\hline $\begin{array}{l}\text { Author (year), } \\
\text { Country }\end{array}$ & $\begin{array}{l}\text { Social relationship } \\
\text { (measures) }\end{array}$ & $\begin{array}{l}\text { Affective } \\
\text { symptoms } \\
\text { (measures) }\end{array}$ & Analysis adjust for & Key findings \\
\hline \multirow[t]{2}{*}{$\begin{array}{l}\text { Popoola \& } \\
\text { Adewuya, } 2012 \\
\text { (Nigeria) }\end{array}$} & \multirow[t]{2}{*}{$\begin{array}{l}\text { Perceived social } \\
\text { support (indicating on } \\
\text { Likert scale) }\end{array}$} & \multirow[t]{2}{*}{$\begin{array}{l}\text { Depression } \\
\text { (MINI) }\end{array}$} & \multirow[t]{2}{*}{ Not reported } & $\begin{array}{l}\text { 1) Depression was } \\
\text { associated with } \\
\text { perceived social support } \\
(p=0.001) \text {. }\end{array}$ \\
\hline & & & & $\begin{array}{l}\text { 2) Perceived poor social } \\
\text { support was a } \\
\text { significant predictor of } \\
\text { depression }(\beta=1.078 \text {, } \\
p=0.014)\end{array}$ \\
\hline \multirow[t]{2}{*}{$\begin{array}{l}\text { Aguado Loi et al., } \\
\text { 2013, (USA) }\end{array}$} & $\begin{array}{l}\text { Social support group } \\
\text { attendance } \\
\text { (Demographic } \\
\text { questionnaire) }\end{array}$ & \multirow[t]{2}{*}{$\begin{array}{l}\text { Depression } \\
\text { (PHQ-9) }\end{array}$} & \multirow[t]{2}{*}{ Not reported } & $\begin{array}{l}\text { 1) Increased depression } \\
\text { was associated with } \\
\text { satisfaction with } \\
\text { family/peer support ( } \beta \\
=-0.42, p<0.01 \text { ). }\end{array}$ \\
\hline & $\begin{array}{l}\text { Satisfaction with social } \\
\text { support (Demographic } \\
\text { questionnaire) }\end{array}$ & & & $\begin{array}{l}\text { 2) The amount of } \\
\text { support from family anc } \\
\text { friends was associated } \\
\text { with depressive } \\
\text { symptoms ( } \beta=-0.36 \text {, } \\
p<0.01) \text {. }\end{array}$ \\
\hline $\begin{array}{l}\text { So et al., 2013, } \\
\text { (China) }\end{array}$ & $\begin{array}{l}\text { Social support (MOS- } \\
\text { SSS) }\end{array}$ & $\begin{array}{l}\text { Anxiety and } \\
\text { Depression } \\
\text { (HADS) }\end{array}$ & None & $\begin{array}{l}\text { Patients showed lower } \\
\text { levels of depression ( } \beta \\
=-0.37, p<0.05) \text { and } \\
\text { anxiety }(\beta=-0.28 \text {, } \\
p<0.05) \text { when they had } \\
\text { greater social support }\end{array}$ \\
\hline \multirow[t]{3}{*}{$\begin{array}{l}\text { Waters et al., 2013, } \\
\text { (USA) }\end{array}$} & \multirow[t]{3}{*}{$\begin{array}{l}\text { Perceived social } \\
\text { support (MOS-SSS) }\end{array}$} & $\begin{array}{l}\text { Emotional } \\
\text { well-being }\end{array}$ & \multirow{3}{*}{$\begin{array}{l}\text { Demographics, } \\
\text { depression history } \\
\text { and trait anxiety, } \\
\text { cancer stage, types } \\
\text { of surgical and } \\
\text { adjuvant } \\
\text { treatments }\end{array}$} & \multirow{2}{*}{$\begin{array}{l}\text { 1) Patients showed } \\
\text { higher worrying about } \\
\text { cancer progression } \\
\text { when they had lower } \\
\text { social support }(r=0.16 \text {, } \\
p<0.05) \text {. }\end{array}$} \\
\hline & & $\begin{array}{l}\text { (RAND 36-Item } \\
\text { Health Survey) }\end{array}$ & & \\
\hline & & $\begin{array}{l}\text { Worry (FACT- } \\
\text { B) }\end{array}$ & & $\begin{array}{l}\text { 2) Patients with higher } \\
\text { social support reported } \\
\text { better emotional well- } \\
\text { being }(\text { Wilks' } \Lambda=10.86 \text {, } \\
F[24,1,320]=2.9 \\
p<.0001)\end{array}$ \\
\hline $\begin{array}{l}\text { Yi \& Kim, 2013, } \\
\text { (Korea) }\end{array}$ & Social support (PRQ-II) & $\begin{array}{l}\text { Depressive } \\
\text { symptom } \\
\text { (CES-D) }\end{array}$ & Not reported & $\begin{array}{l}\text { Patients with low social } \\
\text { support reported higher } \\
\text { depression ( } r=-.585 \text {, } \\
p<.0001) \text {. }\end{array}$ \\
\hline
\end{tabular}




\begin{tabular}{|c|c|c|c|c|}
\hline $\begin{array}{l}\text { Author (year), } \\
\text { Country }\end{array}$ & $\begin{array}{l}\text { Social relationship } \\
\text { (measures) }\end{array}$ & $\begin{array}{l}\text { Affective } \\
\text { symptoms } \\
\text { (measures) }\end{array}$ & Analysis adjust for & Key findings \\
\hline \multirow[t]{5}{*}{$\begin{array}{l}\text { Boinon et al., 2014, } \\
\text { (France) }\end{array}$} & $\begin{array}{l}\text { Perceived social } \\
\text { support }\end{array}$ & $\begin{array}{l}\text { Psychological } \\
\text { distress }\end{array}$ & Not reported & $\begin{array}{l}\text { 1) A higher level of } \\
\text { depressive symptoms }\end{array}$ \\
\hline & (SSQ6) & $\begin{array}{l}\text { (Impact of } \\
\text { Event Scale) }\end{array}$ & & $\begin{array}{l}\text { at T2 (after adjuvant } \\
\text { therapy) was associated } \\
\text { with lower quantity of }\end{array}$ \\
\hline & & $\begin{array}{l}\text { Depressive } \\
\text { symptoms }\end{array}$ & & support \\
\hline & & $\begin{array}{l}\text { (Beck } \\
\text { Depression } \\
\text { Inventory) }\end{array}$ & & $\begin{array}{l}(r=-.20, p<0.05) \text {, } \\
\text { instrumental support }(r \\
=-0.26, p<0.01) \text {, and } \\
\text { informational support at } \\
\text { T1(before adjuvant } \\
\text { therapy) }(r=-0.20 \text {, } \\
\text { p<0.05) }\end{array}$ \\
\hline & & & & $\begin{array}{l}\text { 2) Patients who } \\
\text { perceived a higher } \\
\text { instrumental support at } \\
\text { T1 reported a lower level } \\
\text { of depressive symptoms } \\
(\beta=-0.27, p<0.05) \text { at T2 }\end{array}$ \\
\hline \multirow[t]{2}{*}{$\begin{array}{l}\text { Hasson-Ohayon et } \\
\text { al., 2014, (Israel) }\end{array}$} & Social support (CPASS) & $\begin{array}{l}\text { Psychological } \\
\text { distress (BSI) }\end{array}$ & None & $\begin{array}{l}\text { 1) A significant } \\
\text { association was found } \\
\text { between social support } \\
\text { and depression in } \\
\text { younger patient group ( } \beta \\
=-0.32, p=0.016) \text {. }\end{array}$ \\
\hline & & & & $\begin{array}{l}\text { 2) No significant } \\
\text { association was found } \\
\text { between social support } \\
\text { and depression in older } \\
\text { patient group. }\end{array}$ \\
\hline $\begin{array}{l}\text { Hughes et al., 2014, } \\
\text { (USA) }\end{array}$ & Social support (ESSI) & $\begin{array}{l}\text { Depression } \\
\text { (CES-D) }\end{array}$ & $\begin{array}{l}\text { Demographics, } \\
\text { comorbidities, } \\
\text { cancer stage, time } \\
\text { since treatment }\end{array}$ & $\begin{array}{l}\text { Patients with lower } \\
\text { social support at } \\
\text { T1 (prior to cancer } \\
\text { treatments) experienced } \\
\text { higher level of } \\
\text { depressive symptoms } \\
\text { from T1 to T2 }(6 \text { months } \\
\text { after the completion of } \\
\text { cancer treatments) ( } \beta \\
=-.47, \mathrm{t}(137)=-2.97 \text {, } \\
\mathrm{p}=0.004) \text { than patients } \\
\text { with more social } \\
\text { support. }\end{array}$ \\
\hline \multirow[t]{2}{*}{$\begin{array}{l}\text { Schleife et al., } \\
\text { 2014, (Germany) }\end{array}$} & Social support (VAS) & $\begin{array}{l}\text { Anxiety and } \\
\text { Depression } \\
\text { (HADS) }\end{array}$ & Not reported & $\begin{array}{l}\text { 1) Patients receiving } \\
\text { social support showed } \\
\text { less depression }(r=-0.43 \text {, } \\
p<0.01) \text { as well as } \\
\text { anxiety }(r=-0.36, p<0.01) .\end{array}$ \\
\hline & & & & $\begin{array}{l}\text { 2) Higher social support } \\
\text { decreased mental } \\
\text { distress }(\beta=-0.37 \text {, } \\
p<0.01) \text {. }\end{array}$ \\
\hline
\end{tabular}




\begin{tabular}{|c|c|c|c|c|}
\hline $\begin{array}{l}\text { Author (year), } \\
\text { Country }\end{array}$ & $\begin{array}{l}\text { Social relationship } \\
\text { (measures) }\end{array}$ & $\begin{array}{l}\text { Affective } \\
\text { symptoms } \\
\text { (measures) }\end{array}$ & Analysis adjust for & Key findings \\
\hline $\begin{array}{l}\text { Wang et al., 2014, } \\
\text { (China) }\end{array}$ & $\begin{array}{l}\text { Social support } \\
\text { (SSRS) } \\
\text { Perceived social } \\
\text { support (PSSS) }\end{array}$ & $\begin{array}{l}\text { Depression } \\
\text { (CES-D) } \\
\text { Anxiety } \\
\text { (STAI) }\end{array}$ & Not reported & $\begin{array}{l}\text { Patients with strong } \\
\text { perceived social support } \\
(\beta=-0.29, p<0.01 ; \beta \\
=-0.23, p<0.01) \text { and } \\
\text { objective social support } \\
(\beta=-0.12, p<0.05 ; \beta \\
=-0.14, p<0.05) \text { reported } \\
\text { lower depression as well } \\
\text { as anxiety }\end{array}$ \\
\hline \multirow[t]{2}{*}{$\begin{array}{l}\text { Borstelmann et al., } \\
\text { 2015, (USA) }\end{array}$} & $\begin{array}{l}\text { Perceived social } \\
\text { support } \\
\text { (MOS-SSS) }\end{array}$ & \multirow[t]{2}{*}{$\begin{array}{l}\text { Anxiety } \\
\text { (HADS) }\end{array}$} & \multirow[t]{2}{*}{ Not reported } & $\begin{array}{l}\text { 1) } \\
\text { Unsupported/partnered } \\
\text { patients had higher } \\
\text { anxiety }(p<0.0001)\end{array}$ \\
\hline & $\begin{array}{l}\text { Marital subscale of } \\
\text { Perceive partner } \\
\text { support } \\
\text { (CARES) }\end{array}$ & & & $\begin{array}{l}\text { 2) Patients with lower } \\
\text { social support (OR=0.96, } \\
95 \% \mathrm{Cl}=0.95-0.97) \text { and } \\
\text { unsupported/partnered } \\
\text { (OR=2.09, } 95 \% \mathrm{Cl}=1.34- \\
\text { 3.24) reported higher } \\
\text { anxiety }\end{array}$ \\
\hline \multirow[t]{2}{*}{$\begin{array}{l}\text { Ozkaraman et al., } \\
\text { 2015, (Turkey) }\end{array}$} & \multirow[t]{2}{*}{ Social support (CPSSS) } & \multirow[t]{2}{*}{$\begin{array}{l}\text { Social image } \\
\text { anxiety (SIAS) }\end{array}$} & \multirow[t]{2}{*}{ Not reported } & $\begin{array}{l}\text { 1) Patients } \\
\text { demonstrated higher } \\
\text { anxiety about body } \\
\text { image when they } \\
\text { received support from } \\
\text { the spouse and/or } \\
\text { children, but it was lower } \\
\text { among women who had } \\
\text { support only from } \\
\text { friends (KW=16.20; } \\
p=0.02 \text { ) }\end{array}$ \\
\hline & & & & $\begin{array}{l}\text { 2) Higher anxiety was } \\
\text { associated with } \\
\text { decreasing reliance } \\
\text { support }(r=-0.35 \text {, } \\
p<0.001)\end{array}$ \\
\hline \multirow[t]{4}{*}{$\begin{array}{l}\text { Alfonsson et al., } \\
\text { 2016, (Sweden) }\end{array}$} & \multirow[t]{4}{*}{$\begin{array}{l}\text { Lack of social support } \\
\text { (Self-report } \\
\text { Questionnaire) }\end{array}$} & \multirow[t]{4}{*}{$\begin{array}{l}\text { Anxiety and } \\
\text { Depression } \\
\text { (HADS) }\end{array}$} & \multirow[t]{4}{*}{ Not reported } & $\begin{array}{l}\text { 1) Lack of social } \\
\text { support at T1 (shortly } \\
\text { after diagnosis) } \\
\text { predicted anxiety at } \mathrm{T} 1 \\
(\mathrm{p}<0.001) \text {. }\end{array}$ \\
\hline & & & & $\begin{array}{l}\text { 2) Lack of social } \\
\text { support at T1 and T2 } \\
\text { predicted anxiety at T2 } \\
(3 \text { years after diagnosis) } \\
(p=0.027 ; p=0.020) \text {. }\end{array}$ \\
\hline & & & & $\begin{array}{l}\text { 3) Lack of social } \\
\text { support at T1 predicted } \\
\text { depression at T1 } \\
(p=0.004) \text {. }\end{array}$ \\
\hline & & & & $\begin{array}{l}\text { 4) Lack of social } \\
\text { support at T1 and T2 } \\
\text { predicted depression at } \\
\text { T2 }(p=0.01 ; p=0.002) \text {. }\end{array}$ \\
\hline
\end{tabular}




\begin{tabular}{|c|c|c|c|c|}
\hline $\begin{array}{l}\text { Author (year), } \\
\text { Country }\end{array}$ & $\begin{array}{l}\text { Social relationship } \\
\text { (measures) }\end{array}$ & $\begin{array}{l}\text { Affective } \\
\text { symptoms } \\
\text { (measures) }\end{array}$ & Analysis adjust for & Key findings \\
\hline $\begin{array}{l}\text { Malicka et al., 2016, } \\
\text { (Poland) }\end{array}$ & Social support (BSSS) & $\begin{array}{l}\text { Anxiety (STAI) } \\
\text { Depression } \\
\text { (BDI) }\end{array}$ & Not reported & $\begin{array}{l}\text { No association was } \\
\text { found between social } \\
\text { support and anxiety as } \\
\text { well as depression. }\end{array}$ \\
\hline $\begin{array}{l}\text { Berhili et al., 2017, } \\
\text { (Morocco) }\end{array}$ & $\begin{array}{l}\text { Family support (ask } \\
\text { direct question about } \\
\text { family assistance) }\end{array}$ & $\begin{array}{l}\text { Anxiety and } \\
\text { depression } \\
\text { (HADS) }\end{array}$ & $\begin{array}{l}\text { Demographics, } \\
\text { taking analgesic } \\
\text { and/or anxiolytic } \\
\text { treatment, current } \\
\text { treatment type }\end{array}$ & $\begin{array}{l}\text { Patients demonstrated } \\
\text { psychological distress } \\
\text { when they had lack of } \\
\text { social family support } \\
(p<0.001)\end{array}$ \\
\hline $\begin{array}{l}\text { Fong et al., 2017, } \\
\text { (Canada) }\end{array}$ & $\begin{array}{l}\text { Social Support (MOS- } \\
\text { SSS) }\end{array}$ & $\begin{array}{l}\text { Depressive } \\
\text { symptoms } \\
\text { (CES-D) } \\
\text { Stress } \\
\text { (Perceived } \\
\text { Stress Scale) } \\
\\
\text { Positive and } \\
\text { Negative } \\
\text { Affect } \\
\text { (Positive and } \\
\text { Negative } \\
\text { Affect } \\
\text { Schedule) }\end{array}$ & $\begin{array}{l}\text { Demographics, } \\
\text { cancer stage }\end{array}$ & $\begin{array}{l}\text { 1) Decline in social } \\
\text { support quality } \\
\text { predicted increase in } \\
\text { depression }(p=0.003) \text {, } \\
\text { negative affect }(p=0.05) \text {, } \\
\text { and stress }(\beta=-0.22 \text {, } \\
p=0.01) \text {. } \\
\text { 2) Decreases in social } \\
\text { support quantity ( } \beta \\
=-0.20) \text { predicted } \\
\text { increases in stress. } \\
\text { 3) No association was } \\
\text { found between social } \\
\text { support quantity and } \\
\text { negative and positive } \\
\text { affect. }\end{array}$ \\
\hline $\begin{array}{l}\text { Moon et al., 2017, } \\
\text { (USA) }\end{array}$ & $\begin{array}{l}\text { Receiving } \\
\text { emotional/instrumental } \\
\text { support (counting the } \\
\text { total number of } \\
\text { message containing } \\
\text { emotional/instrumental } \\
\text { support expression) }\end{array}$ & $\begin{array}{l}\text { Depression } \\
\text { (CES-D) }\end{array}$ & $\begin{array}{l}\text { Demographics, } \\
\text { total volume of } \\
\text { message }\end{array}$ & $\begin{array}{l}\text { 1) Patients who received } \\
\text { emotional support by } \\
\text { cancer survivors } \\
\text { demonstrated greater } \\
\text { reduction of depression } \\
(\beta=-0.32, p<0.001) \text {. } \\
\text { 2) No association was } \\
\text { found between receiving } \\
\text { emotional support } \\
\text { provided by other new } \\
\text { patients and the } \\
\text { reduction in depression. }\end{array}$ \\
\hline $\begin{array}{l}\text { Schellekens et al., } \\
\text { 2017, (Canada) }\end{array}$ & $\begin{array}{l}\text { Social support (MOS- } \\
\text { SSS) }\end{array}$ & $\begin{array}{l}\text { Mood } \\
\text { disturbances } \\
\text { (POMS) }\end{array}$ & Not reported & $\begin{array}{l}\text { In MBCR group, patients } \\
\text { with increased social } \\
\text { support reported } \\
\text { changes in their mood } \\
\text { disturbances }(\beta=-0.24 \text {, } \\
p=0.004)\end{array}$ \\
\hline $\begin{array}{l}\text { Su et al., 2017, } \\
\text { (Taiwan) }\end{array}$ & $\begin{array}{l}\text { Family support } \\
\text { (APGAR) }\end{array}$ & $\begin{array}{l}\text { Psychiatric } \\
\text { diagnosis } \\
(\mathrm{MINI})\end{array}$ & Not reported & $\begin{array}{l}\text { Higher family support } \\
\text { was associated with } \\
\text { lower risk for major } \\
\text { depressive disorder ( } \beta= \\
0.87, p<0.05 \text { ). }\end{array}$ \\
\hline
\end{tabular}




\begin{tabular}{|c|c|c|c|c|}
\hline $\begin{array}{l}\text { Author (year), } \\
\text { Country }\end{array}$ & $\begin{array}{l}\text { Social relationship } \\
\text { (measures) }\end{array}$ & $\begin{array}{l}\text { Affective } \\
\text { symptoms } \\
\text { (measures) }\end{array}$ & Analysis adjust for & Key findings \\
\hline $\begin{array}{l}\text { Thompson et al., } \\
\text { 2017, (USA) }\end{array}$ & $\begin{array}{l}\text { Social support } \\
\text { (MOS-SSS) }\end{array}$ & $\begin{array}{l}\text { Depressive } \\
\text { symptoms } \\
\text { (CES-D) }\end{array}$ & $\begin{array}{l}\text { Randomization } \\
\text { assignment, levels } \\
\text { of general health, } \\
\text { depressive } \\
\text { symptoms at } \\
\text { baseline }\end{array}$ & $\begin{array}{l}\text { 1) Patients with lower } \\
\text { initial levels of social } \\
\text { support demonstrated } \\
\text { more severe depressive } \\
\text { symptoms ( } \beta=0.33 \text {, } \\
p<0.001) \\
\text { 2) Patients with lower } \\
\text { baseline social support } \\
(\beta=-0.20, p<0.05) \text { as } \\
\text { well as greater decline in } \\
\text { social support ( } \beta=-0.40 \text {, } \\
p<0.05) \text { over time } \\
\text { demonstrated more } \\
\text { depressive symptoms } \\
\text { over time }\end{array}$ \\
\hline $\begin{array}{l}\text { Tomita et al., 2017, } \\
\text { (Japan) }\end{array}$ & $\begin{array}{l}\text { Social support (MOS- } \\
\text { SSS) }\end{array}$ & $\begin{array}{l}\text { Depressive } \\
\text { symptoms } \\
\text { (CES-D) }\end{array}$ & Not reported & $\begin{array}{l}\text { Higher perceived social } \\
\text { support decreased } \\
\text { depressive symptoms } \\
(\beta=-0.25)\end{array}$ \\
\hline $\begin{array}{l}\text { Bright \& Stanton, } \\
\text { 2018, (USA) }\end{array}$ & $\begin{array}{l}\text { Social support (ISEL- } \\
12 \text { ) }\end{array}$ & $\begin{array}{l}\text { Depression } \\
\text { (CES-D) }\end{array}$ & $\begin{array}{l}\text { Demographics, } \\
\text { medical factors, } \\
\text { number of children }\end{array}$ & $\begin{array}{l}\text { Greater social support at } \\
\text { baseline was associated } \\
\text { with lower depressive } \\
\text { symptoms at } 1 \text { month } \\
\text { after hormone therapy } \\
(\beta=-0.41, p<0.001)\end{array}$ \\
\hline $\begin{array}{l}\text { Schmidt et al., } \\
\text { 2018, (Germany) }\end{array}$ & $\begin{array}{l}\text { Perceived social } \\
\text { support (MSPSS) }\end{array}$ & $\begin{array}{l}\text { Affective } \\
\text { fatigue (FAQ) }\end{array}$ & $\begin{array}{l}\text { Socio- } \\
\text { demographics, } \\
\text { clinical } \\
\text { characteristics }\end{array}$ & $\begin{array}{l}\text { Patients with poor social } \\
\text { support }(p=0.001) \\
\text { demonstrated increased } \\
\text { affective fatigue }\end{array}$ \\
\hline $\begin{array}{l}\text { Escalera et al., } \\
\text { 2019, (USA) }\end{array}$ & $\begin{array}{l}\text { Social support (MOS- } \\
\text { SSS) }\end{array}$ & $\begin{array}{l}\text { Psychological } \\
\text { distress (BSI- } \\
18)\end{array}$ & $\begin{array}{l}\text { Demographics, } \\
\text { time since } \\
\text { diagnosis, } \\
\text { adjuvant breast } \\
\text { cancer treatment, } \\
\text { cancer stage, } \\
\text { history of } \\
\text { depression }\end{array}$ & $\begin{array}{l}\text { 1) Patients } \\
\text { demonstrated fewer } \\
\text { depressive symptoms } \\
\text { when they had } \\
\text { emotional/informational } \\
\text { support }(\beta=-0.17 \text {, } \\
p=0.01) \text {, tangle support } \\
(\beta=-0.12, p=0.03) \text {, } \\
\text { positive social } \\
\text { interaction }(\beta=-0.13 \text {, } \\
p=0.03) \\
2) \text { Tangible support ( } \beta \\
=-0.16, p=0.006) \text {, } \\
\text { affectionate support ( } \beta \\
=-0.21, p=0.001) \text {, and } \\
\text { positive social } \\
\text { interaction }(\beta=-0.14, \\
p=0.02) \text { were negatively } \\
\text { associated with anxiety } \\
\text { symptoms }\end{array}$ \\
\hline $\begin{array}{l}\text { Wondimagegnehu } \\
\text { et al., 2019, } \\
\text { (Ethiopia) }\end{array}$ & $\begin{array}{l}\text { Social support } \\
\text { (MSPSS) }\end{array}$ & $\begin{array}{l}\text { Depression } \\
\text { (PHQ-9) }\end{array}$ & Not reported & $\begin{array}{l}\text { Depressed patients were } \\
\text { found to have lower } \\
\text { social support than non- } \\
\text { depressed women } \\
(p=0.027)\end{array}$ \\
\hline
\end{tabular}




\begin{tabular}{|c|c|c|c|c|}
\hline $\begin{array}{l}\text { Author (year), } \\
\text { Country }\end{array}$ & $\begin{array}{l}\text { Social relationship } \\
\text { (measures) }\end{array}$ & $\begin{array}{l}\text { Affective } \\
\text { symptoms } \\
\text { (measures) }\end{array}$ & Analysis adjust for & Key findings \\
\hline \multirow[t]{2}{*}{$\begin{array}{l}\text { Janowski et al., } \\
\text { 2020, (Poland) }\end{array}$} & \multirow[t]{2}{*}{$\begin{array}{l}\text { Social support } \\
\text { (Disease-related Social } \\
\text { Support Scale) }\end{array}$} & \multirow[t]{2}{*}{$\begin{array}{l}\text { Depression } \\
\text { (BDI) }\end{array}$} & \multirow[t]{2}{*}{ Not reported } & $\begin{array}{l}\text { 1) Women with greater } \\
\text { social support } \\
\text { demonstrated lower } \\
\text { depression than those } \\
\text { with lower social } \\
\text { support }(t=4.08 \text {, } \\
p<0.001)\end{array}$ \\
\hline & & & & $\begin{array}{l}\text { 2) Spiritual support was } \\
\text { a significant predictor of } \\
\text { depressive symptoms } \\
\left(R^{2}=0.27, \beta=-0.52\right. \\
t=-5.01, p<0.001) .\end{array}$ \\
\hline \multicolumn{5}{|c|}{ Social support and /or social constraints } \\
\hline \multirow{4}{*}{$\begin{array}{l}\text { Schmidt \& } \\
\text { Andrykowski } \\
\text { 2004, (USA) }\end{array}$} & \multirow{4}{*}{$\begin{array}{l}\text { Social support } \\
\text { (DUKE-SSQ) } \\
\text { Social constraints } \\
\text { (SCS) }\end{array}$} & \multirow{4}{*}{$\begin{array}{l}\text { Anxiety and } \\
\text { Depression } \\
\text { (HADS) }\end{array}$} & \multirow[t]{4}{*}{ Not reported } & \multirow{3}{*}{$\begin{array}{l}\text { 1) Patients with greater } \\
\text { social support } \\
\text { demonstrated lower } \\
\text { depression }(\beta=-0.23 \text {, } \\
p<0.001)\end{array}$} \\
\hline & & & & \\
\hline & & & & \\
\hline & & & & $\begin{array}{l}\text { 2) Patients with greater } \\
\text { social constraints } \\
\text { demonstrated greater } \\
\text { depression as well as } \\
\text { anxiety }(\beta=0.31 \text {, } \\
p<0.001 ; \beta=0.34 \\
p<0.001)\end{array}$ \\
\hline \multirow[t]{2}{*}{$\begin{array}{l}\text { Wong et al., 2018, } \\
\text { (USA) }\end{array}$} & \multirow[t]{2}{*}{$\begin{array}{l}\text { Social constraints } \\
\text { (Social constraints } \\
\text { scale) } \\
\text { Social support (Chinese } \\
\text { version of MOS-SSS) }\end{array}$} & \multirow[t]{2}{*}{$\begin{array}{l}\text { Depressive } \\
\text { symptoms } \\
\text { (CES-D) }\end{array}$} & \multirow[t]{2}{*}{$\begin{array}{l}\text { Demographic, } \\
\text { medical variables, } \\
\text { cancer stage }\end{array}$} & $\begin{array}{l}\text { 1) The indirect effect of } \\
\text { social constraints on } \\
\text { depressive symptoms } \\
\text { through social support } \\
\text { was significant }(\beta=0.11 \text {, } \\
p<0.01)\end{array}$ \\
\hline & & & & $\begin{array}{l}\text { 2) The direct effect of } \\
\text { social support on } \\
\text { depressive symptoms } \\
\text { was significant ( } \beta \\
=-0.28, p<0.01)\end{array}$ \\
\hline
\end{tabular}




\begin{tabular}{|c|c|c|c|c|}
\hline $\begin{array}{l}\text { Author (year), } \\
\text { Country }\end{array}$ & $\begin{array}{l}\text { Social relationship } \\
\text { (measures) }\end{array}$ & $\begin{array}{l}\text { Affective } \\
\text { symptoms } \\
\text { (measures) }\end{array}$ & Analysis adjust for & Key findings \\
\hline \multirow[t]{3}{*}{$\begin{array}{l}\text { Lally et al., 2019, } \\
\text { (USA) }\end{array}$} & \multirow[t]{3}{*}{ Social constraints } & \multirow[t]{3}{*}{$\begin{array}{l}\text { Depressive } \\
\text { symptoms } \\
\text { (CES-D) }\end{array}$} & \multirow[t]{3}{*}{ No covariates } & $\begin{array}{l}\text { 1) Patients who } \\
\text { perceived social } \\
\text { constraints from } \\
\text { family/friends and } \\
\text { spouse/partner reported } \\
\text { higher depressive } \\
\text { symptoms }\end{array}$ \\
\hline & & & & $\begin{array}{l}\text { 2) Patients who } \\
\text { experienced increased } \\
\text { on family/friends social } \\
\text { constraints reported no } \\
\text { changes in their } \\
\text { depressive symptoms } \\
(p=0.049)\end{array}$ \\
\hline & & & & $\begin{array}{l}\text { 3) Patients who } \\
\text { experienced decreased } \\
\text { family/friends social } \\
\text { constraints reported } \\
\text { decreased depressive } \\
\text { symptoms }(p=0.049)\end{array}$ \\
\hline \multicolumn{5}{|c|}{ Social support and family functioning (family conflict and family stress) } \\
\hline \multirow{4}{*}{$\begin{array}{l}\text { Lueboonthavatchai, } \\
\text { 2007, (Thailand) }\end{array}$} & \multirow{4}{*}{$\begin{array}{l}\text { Social support (SSQ) } \\
\text { Family functioning } \\
\text { (Family relationship } \\
\text { and functioning } \\
\text { questionnaire) }\end{array}$} & \multirow{4}{*}{$\begin{array}{l}\text { Anxiety and } \\
\text { depression } \\
\text { (HADS) }\end{array}$} & \multirow[t]{4}{*}{ Not reported } & \multirow{4}{*}{$\begin{array}{l}\text { 1) Patients' anxiety and } \\
\text { depression were } \\
\text { associated with social } \\
\text { support }(p<0.001) \text { and } \\
\text { family relationship and } \\
\text { functioning ( } p<0.001) \text {. } \\
\text { 2) Poor family } \\
\text { relationship and } \\
\text { functioning was a } \\
\text { predictor of anxiety and } \\
\text { depression }(p<0.05) \text {. }\end{array}$} \\
\hline & & & & \\
\hline & & & & \\
\hline & & & & \\
\hline \multirow{4}{*}{$\begin{array}{l}\text { Mantani et al., } \\
\text { 2007, (Japan) }\end{array}$} & \multirow{4}{*}{$\begin{array}{l}\text { Family functioning } \\
\text { (FAD) }\end{array}$} & Anxiety & \multirow[t]{4}{*}{ Not reported } & \multirow{4}{*}{$\begin{array}{l}\text { Patients demonstrated } \\
\text { higher depression when } \\
\text { they perceived } \\
\text { inappropriate affective } \\
\text { responsiveness among } \\
\text { family members }(\beta= \\
0.59, p<0.01) \text {. }\end{array}$} \\
\hline & & $\begin{array}{l}\text { (Zung self- } \\
\text { rating anxiety } \\
\text { scale) }\end{array}$ & & \\
\hline & & Depression & & \\
\hline & & $\begin{array}{l}\text { (Zung self- } \\
\text { rating } \\
\text { depression } \\
\text { scale) }\end{array}$ & & \\
\hline \multirow[t]{2}{*}{$\begin{array}{l}\text { Ashing-Giwa et al., } \\
\text { 2013, (USA) }\end{array}$} & $\begin{array}{l}\text { Social support (MOS- } \\
\text { social support survey) }\end{array}$ & \multirow[t]{2}{*}{$\begin{array}{l}\text { Depressive } \\
\text { symptom } \\
\text { (CES-D) }\end{array}$} & \multirow[t]{2}{*}{ Not reported } & \multirow{2}{*}{$\begin{array}{l}\text { Patients with low social } \\
\text { support }(r=-.37, p<.01) \\
\text { as well as family stress } \\
\text { ( } r=.522, p=<.01) \text { reported } \\
\text { more depressive } \\
\text { symptoms }\end{array}$} \\
\hline & $\begin{array}{l}\text { Family stress (five- } \\
\text { items from Life Stress } \\
\text { Scale) }\end{array}$ & & & \\
\hline
\end{tabular}




\begin{tabular}{|c|c|c|c|c|}
\hline $\begin{array}{l}\text { Author (year), } \\
\text { Country }\end{array}$ & $\begin{array}{l}\text { Social relationship } \\
\text { (measures) }\end{array}$ & $\begin{array}{l}\text { Affective } \\
\text { symptoms } \\
\text { (measures) }\end{array}$ & Analysis adjust for & Key findings \\
\hline $\begin{array}{l}\text { Segrin et al., 2018, } \\
\text { (USA) }\end{array}$ & $\begin{array}{l}\text { Family conflict (Family } \\
\text { Assessment Device) }\end{array}$ & $\begin{array}{l}\text { Anxiety } \\
\text { (PROMIS- } \\
\text { Anxiety short } \\
\text { form) } \\
\text { Depressive } \\
\text { symptoms } \\
\text { (CES-D) }\end{array}$ & Not reported & $\begin{array}{l}\text { 1) Patients } \\
\text { demonstrated higher } \\
\text { depressive symptoms } \\
\text { when family conflict } \\
\text { was high }(\beta=0.17 \text {, } \\
p<0.01)\end{array}$ \\
\hline \multicolumn{5}{|c|}{ Quality of relationships } \\
\hline $\begin{array}{l}\text { Giese-Davis \& } \\
\text { Hermanson, } \\
\text { 2000, (USA) }\end{array}$ & $\begin{array}{l}\text { Quality of couple's } \\
\text { relationship (FRI): } \\
\text { cohesion, expression, } \\
\text { conflict }\end{array}$ & $\begin{array}{l}\text { Mood } \\
\text { disturbance } \\
\text { (POMS) }\end{array}$ & Income & $\begin{array}{l}\text { Patients demonstrated } \\
\text { lower mood disturbance } \\
\text { when they rated the } \\
\text { relationship } \\
\text { (w/partners) greater in } \\
\text { cohesion-expression ( } \beta \\
=-0.42, p<0.01 \text { ) as well } \\
\text { as greater in conflict ( } \beta \\
=-0.40, p<0.001 \text { ) }\end{array}$ \\
\hline $\begin{array}{l}\text { Manne et al., 2007, } \\
\text { (USA) }\end{array}$ & $\begin{array}{l}\text { Relationship } \\
\text { satisfaction (DAS) }\end{array}$ & $\begin{array}{l}\text { Psychological } \\
\text { distress } \\
\text { (Mental Health } \\
\text { Inventory) }\end{array}$ & $\begin{array}{l}\text { Sociodemographic, } \\
\text { ECOG, surgery } \\
\text { type, functional } \\
\text { impairment, time } \\
\text { since diagnosis, } \\
\text { length of } \\
\text { relationship }\end{array}$ & $\begin{array}{l}\text { Greater patient } \\
\text { relationship satisfaction } \\
\text { was associated with } \\
\text { decreased patients' } \\
\text { psychological distress } \\
(\beta=-0.07, p<0.0001)\end{array}$ \\
\hline \multirow[t]{2}{*}{$\begin{array}{l}\text { Segrin et al., 2007, } \\
\text { (USA) }\end{array}$} & \multirow[t]{2}{*}{$\begin{array}{l}\text { Relationship } \\
\text { satisfaction } \\
\text { (RAS) }\end{array}$} & $\begin{array}{l}\text { Anxiety } \\
\text { (PANAS, SF- } \\
12, \text { ICS, and } \\
\text { GSDS) }\end{array}$ & \multirow[t]{2}{*}{ Not reported } & $\begin{array}{l}\text { 1) No association was } \\
\text { found between patients' } \\
\text { anxiety and her reported } \\
\text { relationship quality }\end{array}$ \\
\hline & & $\begin{array}{l}\text { Depression } \\
\text { (CES-D) }\end{array}$ & & $\begin{array}{l}\text { 2) Higher anxiety was } \\
\text { found in patients when } \\
\text { their partners reported } \\
\text { dissatisfied relationship } \\
\text { quality (T1:r=-0.20, } \\
\text { p<0.05; T2:r=-0.28, } \\
\text { p<0.01; T3:r=-0.27, } \\
\text { p<0.05) }\end{array}$ \\
\hline $\begin{array}{l}\text { Al-Zaben et al., } \\
2015, \\
\text { (Saudi Arabia) }\end{array}$ & $\begin{array}{l}\text { Marital quality } \\
\text { (SPS\&QMI) }\end{array}$ & $\begin{array}{l}\text { Anxiety and } \\
\text { Depression } \\
\text { (HADS) }\end{array}$ & Not reported & $\begin{array}{l}\text { No significant } \\
\text { association was found } \\
\text { of anxiety/depression } \\
\text { with the quality of the } \\
\text { marital relationship }\end{array}$ \\
\hline
\end{tabular}




\begin{tabular}{|c|c|c|c|c|}
\hline $\begin{array}{l}\text { Author (year), } \\
\text { Country }\end{array}$ & $\begin{array}{l}\text { Social relationship } \\
\text { (measures) }\end{array}$ & $\begin{array}{l}\text { Affective } \\
\text { symptoms } \\
\text { (measures) }\end{array}$ & Analysis adjust for & Key findings \\
\hline $\begin{array}{l}\text { Simpson et al., } \\
\text { 2002, (Canada) }\end{array}$ & $\begin{array}{l}\text { Social Integration } \\
\text { (ISSSI) }\end{array}$ & $\begin{array}{l}\text { Mental Health } \\
\text { (SCL\&SCID) } \\
\text { Depression } \\
\text { (BDI) }\end{array}$ & $\begin{array}{l}\text { Age, group } \\
\text { membership, GAF, } \\
\text { BDI, and GSI } \\
\text { scores, baseline } \\
\text { social support } \\
\text { score }\end{array}$ & $\begin{array}{l}\text { 1) Women who had } \\
\text { psychiatric illness } \\
\text { assessed by SCID had } \\
\text { lower social support } \\
\text { ( } p<0.001) \text {. } \\
\text { 2) Social integration } \\
\text { was not a predictor of } \\
\text { the present of } \\
\text { psychiatric illness. } \\
\text { 3) Social integration } \\
\text { (adequacy of close } \\
\text { relationships) was a } \\
\text { predictor of depression } \\
\text { at } 1-y e a r \text { post } \\
\text { intervention ( } \beta=-0.23 \text {, } \\
p<0.01 \text { ). } \\
\text { 4) Social integration } \\
\text { (adequacy of more } \\
\text { distant supports) was a } \\
\text { predictor of global } \\
\text { severity of depression at } \\
1 \text { year post-intervention } \\
(\beta=-0.36, p<0.001) \text {. }\end{array}$ \\
\hline \multicolumn{5}{|c|}{ Both Aspects of Social Relationships } \\
\hline $\begin{array}{l}\text { Brothers \& } \\
\text { Andersen, 2009, } \\
\text { (USA) }\end{array}$ & $\begin{array}{l}\text { Perceived social } \\
\text { support (PSS-F) } \\
\text { Social network index } \\
(\mathrm{SNI}) \\
\text { Presence of significant } \\
\text { other/romantic partner }\end{array}$ & $\begin{array}{l}\text { Depression } \\
\text { (CES-D) }\end{array}$ & $\begin{array}{l}\text { Physical } \\
\text { functioning }\end{array}$ & $\begin{array}{l}\text { 1) Depression was not } \\
\text { associated with } \\
\text { perceived social support } \\
\text { 2) Patients' depression } \\
\text { at both initial and } \\
\text { follow-up was } \\
\text { associated with the } \\
\text { presence of support } \\
\text { person }(r=-0.25, p<0.05 \text {; } \\
r=-0.44, p<0.05)\end{array}$ \\
\hline
\end{tabular}




\begin{tabular}{|c|c|c|c|c|}
\hline $\begin{array}{l}\text { Author (year), } \\
\text { Country }\end{array}$ & $\begin{array}{l}\text { Social relationship } \\
\text { (measures) }\end{array}$ & $\begin{array}{l}\text { Affective } \\
\text { symptoms } \\
\text { (measures) }\end{array}$ & Analysis adjust for & Key findings \\
\hline $\begin{array}{l}\text { Gagliardi et al., } \\
2009 \text {, (Italy) }\end{array}$ & $\begin{array}{l}\text { Social network (Social } \\
\text { Network List) } \\
\text { Social support ( } 1 \text { to } 4 \\
\text { Likert-type scale) }\end{array}$ & $\begin{array}{l}\text { Anxiety } \\
\text { (ASQ) } \\
\text { Depression } \\
\text { (CDQ) }\end{array}$ & Not reported & $\begin{array}{l}\text { 1) Patients } \\
\text { demonstrated lower } \\
\text { anxiety }(r=-0.43, p<0.01) \\
\text { and depression }(r=-0.35 \text {, } \\
p<0.05) \text { when they had } \\
\text { strong informational } \\
\text { support from kins } \\
\text { 2) Patients } \\
\text { demonstrated lower } \\
\text { anxiety when they had } \\
\text { strong emotional } \\
\text { support ( } r=-0.356, \\
p<0.05) \text { from kins } \\
\text { 3) No association was } \\
\text { found between social } \\
\text { network and patients' } \\
\text { anxiety and depression }\end{array}$ \\
\hline $\begin{array}{l}\text { Puigpinos-Riera et } \\
\text { al., 2018, (Spain) }\end{array}$ & $\begin{array}{l}\text { Social network (SNI) } \\
\text { Social support (MOS- } \\
\text { SS) } \\
\text { Co-habitation at home }\end{array}$ & $\begin{array}{l}\text { Anxiety and } \\
\text { depression } \\
\text { (HADS) }\end{array}$ & Not reported & $\begin{array}{l}\text { 1) High risks of } \\
\text { depression and anxiety } \\
\text { were associated with } \\
\text { social isolation }(p=0.00 \text {; } \\
p=0.00) \text { and low social } \\
\text { support ( } p=0.00 ; p=0.00) \\
\text { 2) Living alone was } \\
\text { associated with anxiety } \\
(p=0.011) \text {. }\end{array}$ \\
\hline $\begin{array}{l}\text { Wang et al., 2019, } \\
\text { (USA) }\end{array}$ & $\begin{array}{l}\text { Social support (MOS- } \\
\text { SSS) } \\
\text { Social network index } \\
\text { (count a total number } \\
\text { of people who talk at } \\
\text { least once every two } \\
\text { weeks) }\end{array}$ & $\begin{array}{l}\text { Depression } \\
\text { and anxiety } \\
\text { (PROMIS-short } \\
\text { form) }\end{array}$ & $\begin{array}{l}\text { Demographics, the } \\
\text { level of } \\
\text { acculturation (only } \\
\text { for Chinese } \\
\text { women), and } \\
\text { clinical variables }\end{array}$ & $\begin{array}{l}\text { 1) Patients showed } \\
\text { more depression and } \\
\text { anxiety when they had } \\
\text { less social support (all } \\
\text { p<0.05) } \\
\text { 2) No association was } \\
\text { found between social } \\
\text { network and patients' } \\
\text { anxiety and depression }\end{array}$ \\
\hline
\end{tabular}

\subsection{Functional aspects of social relationships}

Social support, satisfaction of social support, quality of the relationship, social constraints, and family functioning (including family conflict and family stress) are functional social relationships included in this review.

\subsubsection{Social support}

Forty-nine studies examined the association between social support and affective symptoms among breast cancer patients. Of those 49 studies, four did not find any associations of affective symptoms with social support(19-22), whereas one showed that patients' affective symptoms can be changed depending on the source of provided support was.(19) A reduction in patients' depression was reported when patients received peer support from patients who are newly diagnosed with cancer rather than from patients who are undergoing active treatment.(19) 
Among 45 studies that reported significant association with affective symptoms, 33 investigated the association of patients' affective symptoms with the quantity of social support that patients received. The quantity of social support refers to the amount of social support that is available to patients (e.g., frequency of meetings).(23) Patients showed lower levels of anxiety, depression, worry, mood disturbances, and psychological/mental distress when they received a greater quantity of social support. $(12,22,24-46)$ Furthermore, some studies reported that the quantity of social support can predict the levels of patients' affective symptoms including their emotional well-being.(47-54)

In addition to the quantity of social support, seven studies reported an association between type of social support and affective symptoms. Emotional (i.e., subjective) support, defined as support that includes the provision of care, empathy, and trust, was found to be most helpful to decrease patients' depression and anxiety. $(36,46,52,55)$ In other words, as patients received stronger emotional/subjective support, their experience of affective symptoms decreased. Some longitudinal studies showed that emotional/subjective support can function as a predictor of patients' anxiety and depression. $(32,56,57)$ Additionally, improvements in affective symptoms occurred when tangible support such as material support/assistance (e.g., brochures) was provided.(32, 36, 52, 55, 56)

In six studies, patients' affective symptoms were affected by source and satisfaction of social support received in six studies. When patients received support from their family members, including a spouse or children, they reported less anxiety and depression.(41,58-61) However, one study showed less depression and anxiety when support was received from friends compared to support from family.(62) In addition, higher satisfaction with support received was associated with the lower levels of patients' anxiety and depression. $(30,58,63-66)$ A study reported that patients showed less affective symptoms when they were more satisfied with support from family than from friends.(62) Patients' affective symptoms were not related to whether they were satisfied with their friend's support but were related to the amount of support received from a friend.

\subsubsection{Social support and/or social constraints}

Three studies have examined the association of patients' depression with social constraints.(67-69) Patients who perceived social constraints from family (including spouse/partners) or friends showed higher depressive symptoms. However, patients showed lower depression when they had decreased family/friend social constraints. Patients reported no change in depression when social constraints increased. $(68,69)$ Also, lower depression was reported when patients received greater social support.

\subsubsection{Social support and Family functioning}

One study found that both patients' anxiety and depression decreased when they had greater social support and a better-functioning family.(70) Furthermore, family functioning predicted the levels of patients' anxiety and depression. (70) In line with this finding, three other studies also found higher depression in patients when they perceived poor/ineffective family functioning. Specifically, depression greatly increased when patients experienced inappropriate responses from family(71), conflicts between its members (i.e., family conflict)(72), and stress due to the demands on the family (i.e., family stress).(73)

\subsubsection{Quality of relationships}

Four studies investigated the quality of relationships with patients' partners/spouses that patients perceived and assessed its association with their affective symptoms. Of those four, two of them failed to show any significant associations of affective symptoms with the quality of couple/marital relationships. $(74,75)$ However, one study showed that anxiety was not associated with patient's reported relationship quality but with the partner's reported relationship quality.(75) The other two studies showed that patients' psychological distress and mood disturbance increased when patients reported unsatisfying relationships with their spouse/partners.(76, 77$)$ Specifically, one study

Page $30 / 40$ 
found that lower mood disturbance was reported when patients have a partner relationship with greater cohesion and expression (i.e., open communication) as well as more constructive conflicts.(77) The authors interpreted constructive conflicts as an indicator of greater engagement in the relationship with partners. In other words, constructive conflicts can occur due to greater discussion/understanding of each other's specific needs, and this constructive conflict can help reduce patients' mood disturbances.

\subsection{Structural aspects of social relationships}

Structural aspects of social relationships refers to the structure of social networks, such as the size and the linkage between members within a social network.(7) This review included social integration as the structural social relationships (Table 2).

\subsubsection{Social integration}

In this review, one randomized controlled trial that investigated the effect of psychoeducational intervention examined the association between social integration and affective symptoms of breast cancer patients.(78) Social integration did not show any associations with the presence of psychiatric illness. However, one year after psychoeducational intervention, patients showed overall less depression when they perceived adequacy of both close relationships and more distant social ties (i.e., greater social integration).

\subsection{Both aspects of social relationships}

\subsubsection{Social networks}

Social networks can be assessed through whether individuals have important persons in their lives, type (e.g., friends or family) and duration of the relationship, and the frequency of contact with that persons.(79) Four studies assessed both of social networks and social support and their associations with patients' affective symptoms. Patients showed less anxiety and depression when they had stronger social support and social network.(80-83) Specifically, one longitudinal study showed that patients' depression at both initial and follow-up appointments was improved when they had a support person.(80) Another study also reported that living alone (without having a support person) contributed to increased anxiety level.(82)

\section{Discussion}

Sixty-five studies met the inclusion criteria and informed this review. None of the included studies examined the association of social relationships with patients' cognitive symptoms, thus including studies that investigated the association of social relationships with affective symptoms. Of those 65 studies, four studies completely failed to show significant associations of affective symptoms with any aspects of social relationships.; $(20-22,74)$ We found that most patients who participated in those four studies were primarily treated with surgery, which could be interpreted as showing very early stage breast cancer. In contrast/However, in patients with advanced cancer (metastatic disease), social relationships play an important role in promoting their affective symptoms. $(33,60,63,77)$ This finding suggests that patients with advanced stage cancer can benefit from social relationships in managing their affective symptoms compared with those with early stages of cancer.

In this review, social relationships were divided into their function and structural aspects. Functional aspects of social relationships include four variables: social support, social constraints, family functioning, and quality of relationship. Social support refers to aid provided (e.g., emotional or instrumental) through contact with one's social networks (e.g., friends or family), $(7,79)$ whereas social constraints are social conditions that hinder individuals' expression of stressors due to unsupportive, misunderstood, or isolated responses from others.(84) Our findings clearly show that patients' 
affective symptoms can be improved depending on the quantity (e.g., time spent or availability), type (e.g., tangible aid or empathy), source (i.e., who provided support), and satisfaction from the support that they received. Contrary to such positive impact, patients showed greater affective symptoms when they perceived negative social interactions (i.e., social constraints) and poor family functioning. Compared with other functional aspects, findings regarding the quality of social relationships are not consistent. Some studies reported that the quality of relationships is associated with patients' affective symptoms $(76,77)$, but others do not. $(74,75)$ Furthermore, one study showed that patients who reported greater conflicts in relationships with partners also reported lower mood disturbances.(77) Future studies would benefit from ensuring consistency and specificity in defining and measuring quality of social relationships.

Similarly, structural aspects of social relationships also show an association with patients' affective symptoms. All five included studies showed that having a support person, not living alone, and building close relationships with others are factors that lower patients' affective symptoms. It is possible that patients with larger social networks and greater social integration may increase the odds that patients will have friends and family who survive as peer and familiar support.(85) This support can be beneficial while patients are managing symptoms from disease and/or treatment.(85) Additional research is needed to understand how structural and functional aspects of social relationships interact and influence patients' affective symptoms. This understanding may help identify important concepts for models that promote social relationships in breast cancer patients that will help improve their affective symptoms.

\section{Implication for Practice}

Most interventions for those with affective symptoms have primarily focused on managing their internal clinical characteristics. However, our findings reveal that positive social relationships benefit in mitigating affective symptoms of women with breast cancer. Thus, health care providers need to educate patients about the importance of building solid social relationships and encourage them to participate in a supportive network of friends and family members. Patients with advanced cancer may find it highly beneficial to have access to support groups that are relevant to their specific needs. This type of support may help mitigate patients' affective symptoms.

It is also important to assess whether patients have anyone to talk to or attend appointments with them. This type of assessment may be helpful in preventing, and furthermore, mitigating their affective symptoms. Additionally, establishing a social system to support coordination of various types of social relationships from healthcare professionals may yield positive affective outcomes in breast cancer patients. More expanded studies on the impact of social relationships on affective symptoms of breast cancer patients are recommended.

\section{Limitations}

Our study goal was to find literature hat examined the association between social relationships and cognitive symptoms among breast cancer patients. The review of literature yielded that there are no published studies that study this association based on our review criteria. During the literature search, we found several studies that investigated this association in healthy older adults. $(8,9)$ However, no studies have been conducted in the context of breast cancer. We only included articles that explore the association between social relationships and affective symptoms of breast cancer patients. Future research is needed that considers the effect of social relationships on cognitive symptoms in breast cancer is needed to advance our knowledge in cancer symptom science.

Approximately half of the included studies did not report confounding factors (e.g., sociodemographic) and did not adjust for these factors This is an important limitation because the associations between social relationships and a patients' affective symptoms could differ depending on confounding factors. Thus, it is essential to report and adjust for confounding factors using statistical methods. 
Another limitation is that fewer included studies focused on assessing the association between structural aspects of social relationship and patients' affective symptoms. To fully understand the role of structural aspects of social relationships on patient's affective symptoms, further studies are needed that include diverse aspects of social relationships are needed.

Lastly, we found that most included studies used a cross-sectional design, and only a few used Randomized control trials (RCTs) design. To better understand the influence of social relationships on patients' affective symptoms, studies with alternative designs such as longitudinal studies and RCTs are needed. Additionally, most patients included this study were Caucasian women, which could impede generalizability of the study findings. Therefore, a large and heterogeneous sample is needed for future studies to be representative of all women breast cancer patients from all ethnicities.

\section{Conclusions}

This scoping review summarized current evidence concerning social relationships that are associated with affective symptoms of a breast cancer patient. Of the identified social relationships, social support was most identified, followed by social constraints, family functioning, quality of relationships, social networks, and social integration. Our review results support the concept of an association between social relationships and affective symptoms of breast cancer patients, although the specific nature of this association remains unclear. Understanding different aspects of social relationships and their differential effects on patients' affective symptoms will contribute to development of interventions for best practices to support the well-being of this patient population.

\section{Abbreviations}

RCTs= Randomized control trials; MOS-SSS= Medical Outcomes Survey-Social Support Survey; CARES=Cancer Rehabilitation Evaluation System; HADS=Hospital Anxiety and Depression Scale; SSS= Social Support Scale; CES-D= Center for Epidemiological Studies Depression Scale; PRQ-II=Personal Resource Questionnaire II; FACT-B= Functional Assessment of Cancer Therapy-Breast; POMS=Profile of Mood States; POMS-SF=Profile of Mood States-Short Form; NSSQ=Norbeck Social Support Questionnaire;SSQ6=Social Support Questionnaire Short Form; STAI=State-Trait Anxiety Inventory; SSRS=Social Support Rating Scale; PSSS=Perceived Social Support Scale; FSSS=Familiar Social Support Scale; SSDS=Siddiqui-Shah Depression Scale; SCS=Social Constraints Scale; ASQ=Anxiety Scale Questionnaire; $\mathrm{CDQ}=$ Clinical Depression Questionnaire; RCT=Randomized Controlled Trial; MBSR=Mindfulness-Based Stress Reduction; SET=Supportive Expressive Group Therapy; CSOSI=Calgary Symptom of Stress Inventory; PANAS=Positive and Negative Affect Schedule; RAS=Relationship Assessment Scale; MIS=Lewis Mutuality and Interpersonal Sensitivity Scale; FHI=Family Hardiness Index; MDSS=Multi-Dimensional Support Scale; BDI-SF=Beck Depression Inventory-Short Form; DUKE-SSQ=Duke-UNC Functional Social Support Questionnaire; SCS=Social Constraints Scale; FRI=Family Relationship Index; MSPSS=Multidimensional Scale of Perceived Social Support; PHQ-9=Patient Health Questionnaire 9; DAS=Dyadic Adjustment Scale; FACT-B: Functional Assessment of Cancer Therapy-Breast; BSI=Brief Symptom Inventory; CPASS=Cancer Perceived Agents of Social Support; ESSI=ENRICHD Social Support Instrument; ISEL=Support Evaluation List; SCL-90R: Standard Checklist-90-Revised; GSI=Global Severity Index; FAQ=Fatigue Assessment Questionnaire; MINI=Mini International Neuropsychiatric Interview; APGRA=Adaptability, Partnership, Growth, Affection, and Resolve; SNI=Berkman-Syme Network Index; FES=Family Environment Scale; PROMIS=Patient Reported Outcome Measurement Information System; CPSSS=Cancer Patient's Social Support Scale; SIAS=Social Image Anxiety Scale; MIHT=Multidimensional Inventory of Hypochondriacal Traits; USII=Unsupportive Social Interactions Inventory; SSQ= Social Support Questionnaire; TMD=Total Mood Disturbance; FACIT-G=Functional Assessment of Chronic Illness Therapy-General; SPS=Spousal Perception Scale; QMI=Quality of Marriage Index; ISEL-12=Interpersonal Support 
Evaluation List (12 items); SPS-M: Social Provision Scale-Modified; BSSS=Berlin Social Support Scale; SCID=Structured Clinical Interview for DSM-III-R; VAS=Visual Analogue Scales; ISSB=Inventory of Socially Supportive Behaviors; MISSB=Modified Inventory of Socially Supportive Behaviors; SF-12=12-item Short Form Survey; ICS=Index of Clinical Stress; GSDS=General Symptom Distress Scale

\section{Declarations}

Ethics approval and consent to participate: Not applicable.

Consent for publication: Not applicable

Availability of data and material: All data generated or analyzed during this study are included in this published article and its supplementary information files.

Competing interests: The authors declare that they have no conflict of interest.

Funding: No funding was obtained for this study.

Authors' contributions: YY, YL, GS, and TN were involved in the concept and design of the study. LL was involved in literature search. YY, YL, GS, SM, NC, and JZ were involved in reviewing extracted data and interpretation of the findings. YY wrote the first draft of the manuscript, and YL, GS, SM, NC, JZ, LL, and TN contributed to subsequent drafts. All authors have read and approve the manuscript.

Acknowledgments: Not applicable

\section{References}

1. DeSantis CE, Ma J, Gaudet MM, Newman LA, Miller KD, Goding Sauer A, et al. Breast cancer statistics, 2019. CA: A Cancer Journal for Clinicians. 2019;69(6):438-51.

2. DeSantis CE, Ma J, Goding Sauer A, Newman LA, Jemal A. Breast cancer statistics, 2017, racial disparity in mortality by state. CA Cancer J Clin. 2017;67(6):439-48.

3. So WKW, Law BMH, Ng MSN, He X, Chan DNS, Chan CWH, et al. Symptom clusters experienced by breast cancer patients at various treatment stages: A systematic review. Cancer Medicine. 2021;10(8):2531-65.

4. Starkweather AR, Lyon DE, Elswick RK, Jr., Montpetit AJ, Conley Y, McCain NL. A Conceptual Model of Psychoneurological Symptom Cluster Variation in Women with Breast Cancer: Bringing Nursing Research to Personalized Medicine. Curr Pharmacogenomics Person Med. 2013;11(3):224-30.

5. Kim E, Jahan T, Aouizerat BE, Dodd MJ, Cooper BA, Paul SM, et al. Differences in symptom clusters identified using occurrence rates versus symptom severity ratings in patients at the end of radiation therapy. Cancer nursing. 2009;32(6):429-36.

6. Ng T, Dorajoo SR, Cheung YT, Lam YC, Yeo HL, Shwe M, et al. Distinct and heterogeneous trajectories of selfperceived cognitive impairment among Asian breast cancer survivors. Psychooncology. 2018;27(4):1185-92.

7. August KJ, Rook KS. Social Relationships. In: Gellman MD, Turner JR, editors. Encyclopedia of Behavioral Medicine. New York, NY: Springer New York; 2013. p. 1838-42.

8. Kuiper JS, Zuidersma M, Zuidema SU, Burgerhof JG, Stolk RP, Oude Voshaar RC, et al. Social relationships and cognitive decline: a systematic review and meta-analysis of longitudinal cohort studies. Int J Epidemiol. 2016;45(4):1169-206. 
9. Kelly ME, Duff H, Kelly S, McHugh Power JE, Brennan S, Lawlor BA, et al. The impact of social activities, social networks, social support and social relationships on the cognitive functioning of healthy older adults: a systematic review. Syst Rev. 2017;6(1):259.

10. Cohen S. Social relationships and health. Am Psychol. 2004;59(8):676-84.

11. Rivera FI, Guarnaccia PJ, Mulvaney-Day N, Lin JY, Torres M, Alegria M. Family Cohesion and its Relationship to Psychological Distress among Latino Groups. Hisp J Behav Sci. 2008;30(3):357-78.

12. Hughes S, Jaremka LM, Alfano CM, Glaser R, Povoski SP, Lipari AM, et al. Social support predicts inflammation, pain, and depressive symptoms: longitudinal relationships among breast cancer survivors.

Psychoneuroendocrinology. 2014;42:38-44.

13. Kiecolt-Glaser JK, Gouin JP, Hantsoo L. Close relationships, inflammation, and health. Neurosci Biobehav Rev. 2010;35(1):33-8.

14. Leung J, Pachana NA, McLaughlin D. Social support and health-related quality of life in women with breast cancer: a longitudinal study. Psychooncology. 2014;23(9):1014-20.

15. Hinzey A, Gaudier-Diaz MM, Lustberg MB, DeVries AC. Breast cancer and social environment: getting by with a little help from our friends. Breast Cancer Res. 2016;18(1):54.

16. Arksey H, O'Malley L. Scoping Studies: Towards a Methodological Framework. International Journal of Social Research Methodology. 2005;8(1):19-32.

17. Tricco AC, Lillie E, Zarin W, O'Brien KK, Colquhoun H, Levac D, et al. PRISMA Extension for Scoping Reviews (PRISMA-ScR): Checklist and ExplanationThe PRISMA-ScR Statement. Annals of Internal Medicine. 2018;169(7):467-73.

18. Vardy J, Rourke S, Tannock IF. Evaluation of cognitive function associated with chemotherapy: a review of published studies and recommendations for future research. J Clin Oncol. 2007;25(17):2455-63.

19. Moon T-J, Chih M-Y, Shah DV, Yoo W, Gustafson DH. Breast Cancer Survivors' Contribution to Psychosocial Adjustment of Newly Diagnosed Breast Cancer Patients in a Computer-Mediated Social Support Group. Journalism \& Mass Communication Quarterly. 2017;94(2):486-514.

20. Palesh OG, Shaffer T, Larson J, Edsall S, Chen XH, Koopman C, et al. Emotional self-efficacy, stressful life events, and satisfaction with social support in relation to mood disturbance among women living with breast cancer in rural communities. Breast J. 2006;12(2):123-9.

21. Malicka I, Kozłowska A, Woźniewski M, Rymaszewska J, Szczepańska-Gieracha J. The role of social support in women's health and recovery processes. Psychol Health Med. 2016;21(1):81-91.

22. Friedman LC, Kalidas M, Elledge R, Chang J, Romero C, Husain I, et al. Optimism, social support and psychosocial functioning among women with breast cancer. Psychooncology. 2006;15(7):595-603.

23. Fiorillo D, Sabatini F. Quality and quantity: the role of social interactions in self-reported individual health. Soc Sci Med. 2011;73(11):1644-52.

24. Lee EH, Yae Chung B, Boog Park H, Hong Chun K. Relationships of mood disturbance and social support to symptom experience in Korean women with breast cancer. J Pain Symptom Manage. 2004;27(5):425-33.

25. Talley A, Molix L, Schlegel RJ, Bettencourt A. The influence of breast cancer survivors' perceived partner social support and need satisfaction on depressive symptoms: a longitudinal analysis. Psychol Health. 2010;25(4):43349.

26. Jones SL, Hadjistavropoulos HD, Sherry SB. Health anxiety in women with early-stage breast cancer: What is the relationship to social support? Canadian Journal of Behavioural Science / Revue canadienne des sciences du comportement. 2012;44(2):108-16. 
27. Mallinckrodt B, Armer JM, Heppner PP. A threshold model of social support, adjustment, and distress after breast cancer treatment. J Couns Psychol. 2012;59(1):150-60.

28. Popoola AO, Adewuya AO. Prevalence and correlates of depressive disorders in outpatients with breast cancer in Lagos, Nigeria. Psychooncology. 2012;21(6):675-9.

29. Waters EA, Liu Y, Schootman M, Jeffe DB. Worry about cancer progression and low perceived social support: implications for quality of life among early-stage breast cancer patients. Ann Behav Med. 2013;45(1):57-68.

30. Aguado Loi CX, Baldwin JA, McDermott RJ, McMillan S, Martinez Tyson D, Yampolskaya S, et al. Risk factors associated with increased depressive symptoms among Latinas diagnosed with breast cancer within 5 years of survivorship. Psychooncology. 2013;22(12):2779-88.

31. Yi M, Kim J. Factors influencing health-promoting behaviors in Korean breast cancer survivors. Eur J Oncol Nurs. 2013;17(2):138-45.

32. Boinon D, Sultan S, Charles C, Stulz A, Guillemeau C, Delaloge S, et al. Changes in psychological adjustment over the course of treatment for breast cancer: the predictive role of social sharing and social support. Psychooncology. 2014;23(3):291-8.

33. Hasson-Ohayon I, Goldzweig G, Dorfman C, Uziely B. Hope and social support utilisation among different age groups of women with breast cancer and their spouses. Psychol Health. 2014;29(11):1303-19.

34. Schleife H, Sachtleben C, Finck Barboza C, Singer S, Hinz A. Anxiety, depression, and quality of life in German ambulatory breast cancer patients. Breast Cancer. 2014;21(2):208-13.

35. So WK, Leung DY, Ho SS, Lai ET, Sit JW, Chan CW. Associations between social support, prevalent symptoms and health-related quality of life in Chinese women undergoing treatment for breast cancer: a cross-sectional study using structural equation modelling. Eur J Oncol Nurs. 2013;17(4):442-8.

36. Wang F, Liu J, Liu L, Wang F, Ma Z, Gao D, et al. The status and correlates of depression and anxiety among breastcancer survivors in Eastern China: a population-based, cross-sectional case-control study. BMC Public Health. 2014;14:326.

37. Borstelmann NA, Rosenberg SM, Ruddy KJ, Tamimi RM, Gelber S, Schapira L, et al. Partner support and anxiety in young women with breast cancer. Psychooncology. 2015;24(12):1679-85.

38. Berhili S, Kadiri S, Bouziane A, Aissa A, Marnouche E, Ogandaga E, et al. Associated factors with psychological distress in Moroccan breast cancer patients: A cross-sectional study. Breast. 2017;31:26-33.

39. Schellekens MPJ, Tamagawa R, Labelle LE, Speca M, Stephen J, Drysdale E, et al. Mindfulness-Based Cancer Recovery (MBCR) versus Supportive Expressive Group Therapy (SET) for distressed breast cancer survivors: evaluating mindfulness and social support as mediators. J Behav Med. 2017;40(3):414-22.

40. Schmidt ME, Wiskemann J, Schneeweiss A, Potthoff K, Ulrich CM, Steindorf K. Determinants of physical, affective, and cognitive fatigue during breast cancer therapy and 12 months follow-up. Int J Cancer. 2018;142(6):1148-57.

41. Su JA, Yeh DC, Chang CC, Lin TC, Lai CH, Hu PY, et al. Depression and family support in breast cancer patients. Neuropsychiatr Dis Treat. 2017;13:2389-96.

42. Thompson T, Pérez M, Kreuter M, Margenthaler J, Colditz G, Jeffe DB. Perceived social support in African American breast cancer patients: Predictors and effects. Soc Sci Med. 2017;192:134-42.

43. Tomita M, Takahashi M, Tagaya N, Kakuta M, Kai I, Muto T. Structural equation modeling of the relationship between posttraumatic growth and psychosocial factors in women with breast cancer. Psychooncology. 2017;26(8):1198-204.

44. Bright EE, Stanton AL. Prospective investigation of social support, coping, and depressive symptoms: A model of adherence to endocrine therapy among women with breast cancer. J Consult Clin Psychol. 2018;86(3):242-53. 
45. Wondimagegnehu A, Abebe W, Abraha A, Teferra S. Depression and social support among breast cancer patients in Addis Ababa, Ethiopia. BMC Cancer. 2019;19(1):836.

46. Janowski K, Tatala M, Jedynak T, Wałachowska K. Social support and psychosocial functioning in women after mastectomy. Palliat Support Care. 2020;18(3):314-21.

47. Kim Y, Morrow GR. The effects of family support, anxiety, and post-treatment nausea on the development of anticipatory nausea: a latent growth model. J Pain Symptom Manage. 2007;34(3):265-76.

48. Cohen M, Mabjish AA, Zidan J. Comparison of Arab breast cancer survivors and healthy controls for spousal relationship, body image, and emotional distress. Qual Life Res. 2011;20(2):191-8.

49. Gorman JR, Malcarne VL, Roesch SC, Madlensky L, Pierce JP. Depressive symptoms among young breast cancer survivors: the importance of reproductive concerns. Breast Cancer Res Treat. 2010;123(2):477-85.

50. Kim J, Han JY, Shaw B, McTavish F, Gustafson D. The roles of social support and coping strategies in predicting breast cancer patients' emotional well-being: testing mediation and moderation models. J Health Psychol. 2010;15(4):543-52.

51. Hill J, Holcombe C, Clark L, Boothby MR, Hincks A, Fisher J, et al. Predictors of onset of depression and anxiety in the year after diagnosis of breast cancer. Psychol Med. 2011;41(7):1429-36.

52. Liu DG, Wang SS, Peng RJ, Qin T, Shi YX, Teng XY, et al. Interaction of social support and psychological stress on anxiety and depressive symptoms in breast cancer patients. Asian Pac J Cancer Prev. 2011;12(10):2523-9.

53. Alfonsson S, Olsson E, Hursti T, Lundh MH, Johansson B. Socio-demographic and clinical variables associated with psychological distress 1 and 3 years after breast cancer diagnosis. Support Care Cancer. 2016;24(9):4017-23.

54. Fong AJ, Scarapicchia TMF, McDonough MH, Wrosch C, Sabiston CM. Changes in social support predict emotional well-being in breast cancer survivors. Psychooncology. 2017;26(5):664-71.

55. Escalera C, Santoyo-Olsson J, Stewart AL, Ortiz C, Nápoles AM. Social support as a moderator in the relationship between intrusive thoughts and anxiety among Spanish-speaking Latinas with breast cancer. Psychooncology. 2019;28(9):1819-28.

56. Von Ah D, Kang DH. Correlates of mood disturbance in women with breast cancer: patterns over time. Journal of advanced nursing. 2008;61(6):676-89.

57. Lee MK, Park S, Lee ES, Ro J, Kang HS, Shin KH, et al. Social support and depressive mood 1 year after diagnosis of breast cancer compared with the general female population: a prospective cohort study. Support Care Cancer. 2011;19(9):1379-92.

58. Neuling SJ, Winefield HR. Social support and recovery after surgery for breast cancer: frequency and correlates of supportive behaviours by family, friends and surgeon. Soc Sci Med. 1988;27(4):385-92.

59. Maly RC, Umezawa Y, Leake B, Silliman RA. Mental health outcomes in older women with breast cancer: impact of perceived family support and adjustment. Psychooncology. 2005;14(7):535-45.

60. Hasson-Ohayon I, Goldzweig G, Braun M, Galinsky D. Women with advanced breast cancer and their spouses: diversity of support and psychological distress. Psychooncology. 2010;19(11):1195-204.

61. Ozkaraman A, Culha I, Fadiloglu ZC, Kosgeroglu N, Gokce S, Alparslan GB. Relationships between social support and social image concerns in Turkish women with breast cancer. Asian Pac J Cancer Prev. 2015;16(5):1795-802.

62. Roberts CS, Cox CE, Shannon VJ, Wells NL. A closer look at social support as a moderator of stress in breast cancer. Health Soc Work. 1994;19(3):157-64.

63. Koopman C, Hermanson K, Diamond S, Angell K, Spiegel D. Social support, life stress, pain and emotional adjustment to advanced breast cancer. Psychooncology. 1998;7(2):101-11.

Page $37 / 40$ 
64. Porter LS, Clayton MF, Belyea M, Mishel M, Gil KM, Germino BB. Predicting negative mood state and personal growth in African American and White long-term breast cancer survivors. Ann Behav Med. 2006;31(3):195-204.

65. Gellaitry G, Peters K, Bloomfield D, Horne R. Narrowing the gap: the effects of an expressive writing intervention on perceptions of actual and ideal emotional support in women who have completed treatment for early stage breast cancer. Psychooncology. 2010;19(1):77-84.

66. Boinon D, Sultan S, Charles C, Rosberger Z, Delaloge S, Dauchy S. How social sharing and social support explain distress in breast cancer after surgery: the role of alexithymia. J Psychosoc Oncol. 2012;30(5):573-92.

67. Lally RM, Kupzyk K, Mills A, Gallo S, Meneses K. Effects of social constraints and web-based psychoeducation on cancer-related psychological adjustment early-after breast cancer diagnosis. Journal of Psychosocial Oncology. 2019;37(6):677-98.

68. Wong CCY, Warmoth K, Ivy S, Cheung B, Lu Q. Relation of social constraints on disclosure to adjustment among Chinese American cancer survivors: A multiprocesses approach. Psychooncology. 2018;27(3):977-82.

69. Schmidt JE, Andrykowski MA. The role of social and dispositional variables associated with emotional processing in adjustment to breast cancer: an internet-based study. Health Psychol. 2004;23(3):259-66.

70. Lueboonthavatchai P. Prevalence and psychosocial factors of anxiety and depression in breast cancer patients. J Med Assoc Thai. 2007;90(10):2164-74.

71. Mantani T, Saeki T, Inoue S, Okamura H, Daino M, Kataoka T, et al. Factors related to anxiety and depression in women with breast cancer and their husbands: role of alexithymia and family functioning. Support Care Cancer. 2007;15(7):859-68.

72. Segrin C, Badger TA, Sikorskii A, Crane TE, Pace TWW. A dyadic analysis of stress processes in Latinas with breast cancer and their family caregivers. Psychooncology. 2018;27(3):838-46.

73. Ashing-Giwa K, Rosales M, Lai L, Weitzel J. Depressive symptomatology among Latina breast cancer survivors. Psychooncology. 2013;22(4):845-53.

74. Al-Zaben FN, Sehlo MG, Koenig HG. A cross-sectional study of anxiety and marital quality among women with breast cancer at a university clinic in western Saudi Arabia. Saudi Med J. 2015;36(10):1168-75.

75. Segrin C, Badger T, Dorros SM, Meek P, Lopez AM. Interdependent anxiety and psychological distress in women with breast cancer and their partners. Psychooncology. 2007;16(7):634-43.

76. Manne SL, Norton TR, Ostroff JS, Winkel G, Fox K, Grana G. Protective buffering and psychological distress among couples coping with breast cancer: The moderating role of relationship satisfaction. J Fam Psychol. 2007;21(3):380-8.

77. Giese-Davis J, Hermanson K, Koopman C, Weibel D, Spiegel D. Quality of couples' relationship and adjustment to metastatic breast cancer. J Fam Psychol. 2000;14(2):251-66.

78. Simpson JS, Carlson LE, Beck CA, Patten S. Effects of a brief intervention on social support and psychiatric morbidity in breast cancer patients. Psychooncology. 2002;11(4):282-94.

79. Berkman LF, Glass T, Brissette I, Seeman TE. From social integration to health: Durkheim in the new millennium. Soc Sci Med. 2000;51(6):843-57.

80. Brothers BM, Andersen BL. Hopelessness as a predictor of depressive symptoms for breast cancer patients coping with recurrence. Psychooncology. 2009;18(3):267-75.

81. Gagliardi C, Vespa A, Papa R, Mariotti C, Cascinu S, Rossini S. Social support networks and depression of women suffering from early-stage breast cancer: a case control study. J Psychosoc Oncol. 2009;27(2):216-29.

82. Puigpinós-Riera R, Graells-Sans A, Serral G, Continente $X$, Bargalló X, Domènech $M$, et al. Anxiety and depression in women with breast cancer: Social and clinical determinants and influence of the social network and social support 
(DAMA cohort). Cancer Epidemiol. 2018;55:123-9.

83. Wang JH, Gomez SL, Brown RL, Davis K, Allen L, Huang E, et al. Factors associated with Chinese American and White cancer survivors' physical and psychological functioning. Health Psychol. 2019;38(5):455-65.

84. Lepore SJ, Revenson TA. Social constraints on disclosure and adjustment to cancer. Social and Personality Psychology Compass. 2007;1(1):313-33.

85. Kroenke CH, Kwan ML, Neugut Al, Ergas IJ, Wright JD, Caan BJ, et al. Social networks, social support mechanisms, and quality of life after breast cancer diagnosis. Breast Cancer Res Treat. 2013;139(2):515-27.

\section{Figures}
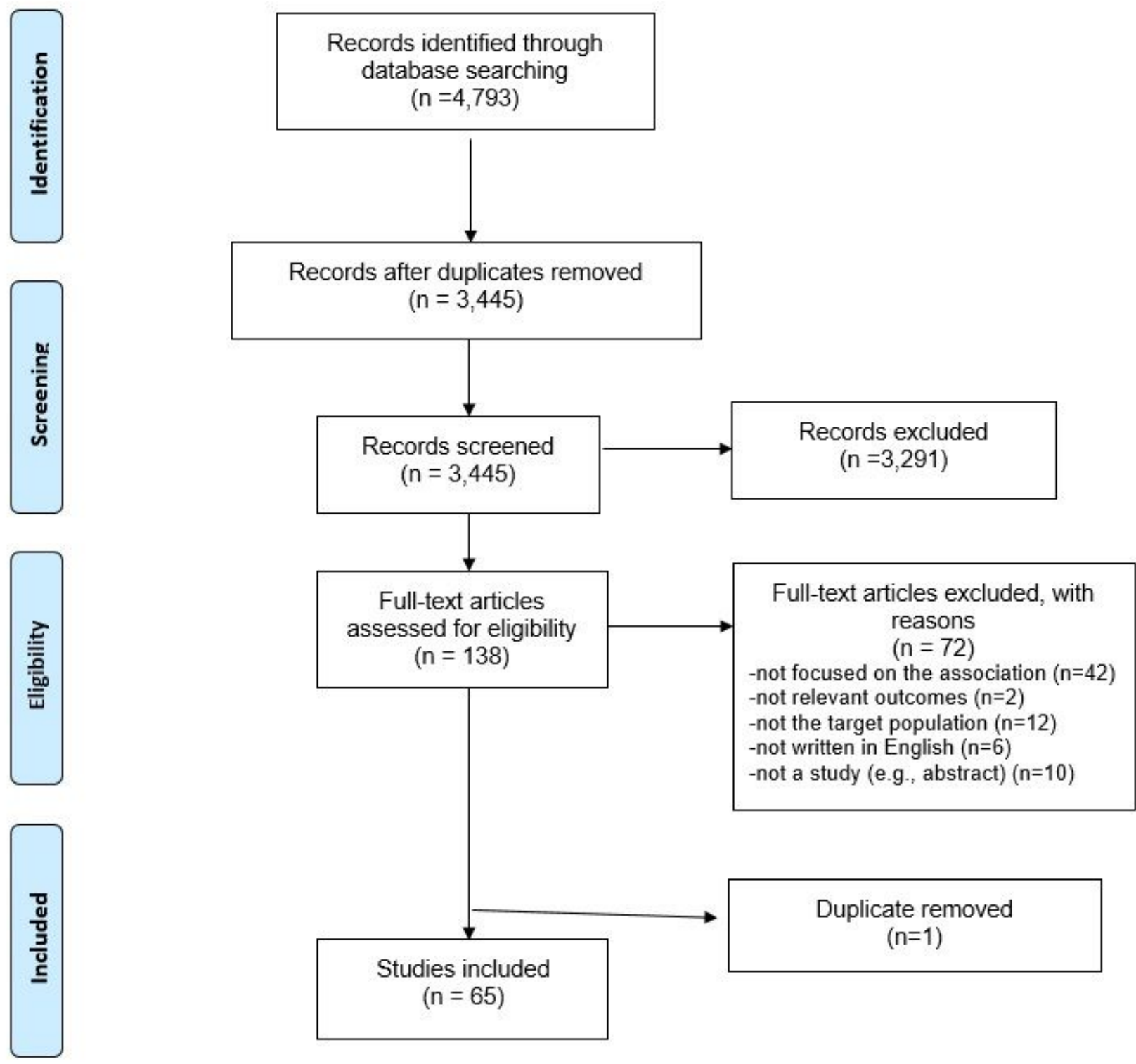

Figure 1

PRISMA-ScR flow diagram for the study selection process

\section{Supplementary Files}


This is a list of supplementary files associated with this preprint. Click to download.

- AppendixABMCCancer.docx 